\title{
Effect of decreasing blockage ratio on DDT in small channels with obstacles
}

\author{
G.B. Goodwin, ${ }^{*}$ R.W. Houim, E.S. Oran \\ Department of Aerospace Engineering, University of Maryland, \\ College Park, Maryland, 20742, USA \\ *Corresponding author: Gabriel Goodwin, ggoodwin@umd.edu, (410)330-9676
}

\begin{abstract}
Multidimensional numerical simulations of an unconfined, homogeneous, chemically reactive gas were used to catalog interactions leading to the deflagration-to-detonation transition (DDT). The configuration studied was an infinitely long rectangular channel with regularly spaced obstacles containing a stoichiometric mixture of ethylene and oxygen, initially at atmospheric conditions and ignited in a corner with a small flame. The channel height is kept constant at $3200 \mu \mathrm{m}$ and obstacle heights varied from $2560 \mu \mathrm{m}$ to $160 \mu \mathrm{m}$ to decrease the blockage ratio $(b r)$ from 0.8 to 0.05 . The compressible reactive Navier-Stokes equations were solved by a high-order numerical algorithm on a locally adapting grid. The initially laminar flame develops into a turbulent flame with the creation of shocks, shock-flame interactions, shock-boundary layer interactions, a host of fluid and chemical-fluid instabilities, and DDT. Several different DDT mechanisms are observed as the $b r$ is reduced. For $b r$ in the range of 0.5 to 0.35, the shocks in the unburned material diffract over the obstacles and reflect against the channel wall, forming Mach stems that increase in strength with every obstacle traversed. Eventually, the Mach stem strength is sufficient for the unburned mixture to detonate after it reflects from an obstacle. For br outside of this range, DDT may occur either through Mach-stem reflection or through direct initiation due to shock focusing. Stochasticity of the turbulence leading to DDT in channels with low br is considered.
\end{abstract}

Keywords: DDT; Turbulent flame; Shock interactions; Direct initiation; Numerical simulations

\section{Introduction}

Deflagration-to-detonation transition (DDT) is a complex process involving turbulence, shock collisions and reflections, shock-flame interactions, and interactions of shocks with boundary layers. It is an important topic because in many cases DDT must be prevented $[1,2]$ and in others its occurence must be controlled in time and space [3-5]. DDT may be initiated by a number of different mechanisms that have been seen in both computations and experiments [6-15]. Because of the complexity of the flow, not all of these mechanisms, or the interplay among them, are completely understood. 
Direct initiation of a detonation in a gas has been studied experimentally and theoretically [6-

8]. Sufficiently large external energy addition at a timescale that is small relative to the acoustic timescale of the explosive mixture can result in a direct initiation of a detonation without first creating a measurable deflagration [16]. Recently, the response of the inert gas to a localized, transient energy deposition has been studied by asymptotic analysis in an effort to quantify the gas response (deflagration or detonation) [17]. Initiation of a detonation through a gradient of chemical reactivity [18, 19] has been studied extensively using numerical simulations, as summarized in [10]. DDT in channels with obstacles has been simulated for a variety of premixed fuels [11-13] and has also been the subject of experimentation [20].

The effect of obstacles, often characterized by a blockage ratio $(b r$, or the obstacle height divided by the channel height), on flame acceleration and DDT has been investigated numerically [21] and experimentally [14, 15]. Gamezo et al. [21] noted the competing effects of high blockage ratios: larger obstacles promote nonuniform flow, which leads to rapid flame acceleration, and they also weaken shocks diffracting over large obstacles. These authors also observed that the location in the channel where DDT occurs does not vary significantly for the $b r=0.31-0.56$ range, and that distance into the channel where DDT occurs increases sharply outside of this interval for both higher and lower $b r$.

This paper presents a series of simulations covering the range $b r=0.05-0.8$ in order to identify how the mechanism of DDT changes as blockage ratio is varied. We will discuss the transition from an initially laminar flame to a turbulent flame and DDT for several different regimes of flow characterized by $b r$. DDT in the high $b r$ regime $(0.35<b r<0.8)$ occured in gradients of reactivity formed behind Mach-stem reflections against obstacles through the gradient mechanism. In the intermediate regime $(0.05<b r<0.35)$, DDT occurred either behind a Mach reflection, at a flame front, or in a volume of unburned gas subjected to shock focusing through a direct initiation mechanism. In the low $b r$ regime (0.05), the DDT location was against an obstacle or channel wall. In the case of $b r=0.8, \mathrm{DDT}$ occurs due to both mechanisms, shock focusing through direct initiation and detonation through a gradient mechanism.

\section{Background}

DDT in channels was first observed in multidimensional simulations when researchers wanted to determine what was required to simulate DDT computationally [10-13]. The expected result was for the flame front to transition to a detonation as it accelerated down the channel, but researchers were surprised to see the detonation initiate in the unburned mixture between a leading shock and the turbulent flame front. This region between the leading shock and flame was shock-heated, with significantly 
increased temperature, pressure and density.

DDT ignition in these early simulations was consistent with the DDT mechanism first observed in calculations by Zeldovich et al. [18], who computed the one-dimensional (1D) propagation of a chemical reaction in a detonable mixture, using several different initial temperature gradients. Different regimes of chemical reaction development were observed. For steep initial temperature gradients, a weak shock formed that quickly separated from the reaction front and the reaction proceeded as a normal flame propagation. Conversely, for very small initial temperature gradients, the reaction proceeded throughout the entire mixture uniformly and a shock did not form. In an intermediate range of temperature gradients, the shock wave coupled with the reaction front. The chemical reaction intensified the shock strength, eventually forming an overdriven detonation wave that gradually decayed to the Chapman-Jouguet velocity, $D_{C J}$. This DDT ignition-gradient mechanism was later observed in experiments conducted by Lee et al. [19]. Detonable mixtures were ignited through photochemical initiation and it was determined that detonation initiation required a minimum peak value of free radical concentration as well as an appropriate gradient of the free radical distribution. If the mixture's gradient of reactivity supported propagation of the chemical reaction in phase with the shock propagation, the coherent energy release gave rise to a rapid amplification of the shock, leading to the formation of a detonation. Lee et al. referred to this phenomenon as the "Shock Wave Amplification by Coherent Energy Release" (SWACER) mechanism.

The effect of obstacles in the flow on flame acceleration and DDT in channels has been researched extensively [10-12]. Using obstacles with a $b r=0.5$ was the predominant method for accelerating the flame and producing strong shocks ahead of the flame in the unburned mixture. The shocks diffracted over the obstacles and formed Mach stems after reflecting from the channel wall, with the strength of the Mach stem increasing with each obstacle traversed. Collision of the Mach stems with the obstacles greatly increased the temperature of the unburned mixture and created a gradient of reactivity. When a correct gradient of reactivity formed such that the temperature reached the autoignition threshold for the mixture, a spontaneous wave would emerge with speed $D_{s p}[10]$,

$$
D_{s p}=-\frac{\nabla \tau_{c}}{\left|\nabla \tau_{c}\right|^{2}}
$$

where $\tau_{c}$ is induction time. This spontaneous wave propagated through the gradient of reactivity and became an overdriven detonation. DDT ignition in channels with obstacles was consistent with the mechanisms observed in earlier work $[18,19]$.

In channels with smaller $b r$, the diffraction of the shocks over the obstacles does not produce the strong Mach stems seen in the channels with larger $b r$. Recent numerical simulations [22] have shown 
that the shock heated, unburned mixture may detonate due to shock reflections and collisions rapidly depositing energy into a localized volume at a timescale smaller than the acoustic timescale of the gas. In this regime, DDT does not occur as predictably as it does in the high $b r$ regime when it forms in a gradient of reactivity against an obstacle. DDT occurs wherever the rate of energy deposition into a volume of the unburned mixture is sufficient to directly initiate a detonation. This may happen when multiple shocks reflect against the obstacles and channel walls, then collide with one another, or even when shocks intersect in unobstructed space. The collision deposits sufficient energy into the unburned mixture to initiate a detonation. Experimentally, this has been shown to occur against or near the channel wall, just after the flame has traversed an obstacle, likely due to the same energy deposition mechanism [23]. This paper explores these DDT mechanisms observed in a series of two-dimensional (2D) and three-dimensional (3D) calculations with varying $b r$.

\section{Numerical and Physical Model}

The numerical model solves the full set of Navier-Stokes equations for an unsteady, fully compressible, chemically reacting gas, an ideal gas equation of state, and a simplified, calibrated chemical-diffusive model is used to convert fuel to product,

$$
\begin{gathered}
\frac{\partial \rho}{\partial t}+\nabla \cdot(\rho \mathbf{U})=0 \\
\frac{\partial(\rho \mathbf{U})}{\partial t}+\nabla \cdot(\rho \mathbf{U} \mathbf{U})+\nabla P=\nabla \cdot \hat{\tau} \\
\frac{\partial E}{\partial t}+\nabla \cdot((E+P) \mathbf{U})=\nabla \cdot(\mathbf{U} \cdot \hat{\tau})+\nabla \cdot(K \nabla T)-\rho q \dot{w} \\
\frac{\partial(\rho Y)}{\partial t}+\nabla \cdot(\rho Y \mathbf{U})+\nabla \cdot(\rho D \nabla Y)-\rho \dot{w}=0 \\
P=\frac{\rho R T}{M} \\
\frac{d Y}{d t} \equiv \dot{w}=-A \rho Y \exp \left(-E_{\mathrm{a}} / R T\right)
\end{gathered}
$$

where $\rho$ is the mass density, $\mathbf{U}$ is the velocity, $E$ is the energy density, $P$ is the pressure, $T$ is the temperature, $Y$ is the mass fraction of reactant, $\dot{w}$ is the reaction rate, $q$ is the total chemical energy release, $K$ is the thermal conduction coefficient, $D$ is the mass diffusion coefficient, $R$ is the universal gas constant, $M$ is the molecular weight, $A$ is the pre-exponential factor, and $E_{\mathrm{a}}$ is the activation energy. Thermal conductivity, $\kappa$, viscosity, $\nu$, and mass diffusivity, $D$, were modeled using reference values of $1.0 \times 10^{-5} \mathrm{~g} / \mathrm{s}-\mathrm{cm}-\mathrm{K}^{0.7}, 7.0 \times 10^{-6} \mathrm{~g} / \mathrm{s}-\mathrm{cm}-\mathrm{K}^{0.7}$, and $1.0 \times 10^{-5} \mathrm{~g} / \mathrm{s}-\mathrm{cm}-\mathrm{K}^{0.7}$, respectively. The viscous stress tensor is defined as 


$$
\hat{\tau}=\rho \nu\left((\nabla \mathbf{U})-(\nabla \mathbf{U})^{T}-\frac{2}{3}(\nabla \cdot \mathbf{U}) \mathbf{I}\right),
$$

where $\mathbf{I}$ is the unit tensor and the superscript $T$ indicates matrix transposition. A Godunov algorithm, fifth-order accurate in space and third-order accurate in time [24], is used to solve the equations on a dynamically adapting mesh [25]. Obstacles were modeled using an immersed boundary method [26]. The simulations described below used meshes with a minimum spacing $d x_{\min }=3.3 \mu \mathrm{m}$ and coarsest spacing of $d x_{\max }=53.3 \mu \mathrm{m}$. The largest grid had 60 cells across the channel height and the smallest had 960 cells. This choice of grids was tested and shown to resolve the flames, boundary layers, shocks, and other important flow and chemical structures.

Table 1. Input model parameters and output combustion wave properties for stoichiometric ethylene and oxygen initially at 1 atm and $298 \mathrm{~K}$.

\begin{tabular}{|c|c|c|}
\hline \multicolumn{3}{|c|}{ Input } \\
\hline$P_{\mathrm{O}}$ & 1 atm & Initial Pressure \\
\hline$T_{\mathrm{O}}$ & $298 \mathrm{~K}$ & Initial Temperature \\
\hline$\rho_{\mathrm{O}}$ & $1.27 \times 10^{-3} \mathrm{~g} / \mathrm{cm}^{3}$ & Initial Density \\
\hline$\gamma$ & 1.2195 & Adiabatic Index \\
\hline$M$ & $31 \mathrm{~g} / \mathrm{mol}$ & Molecular Weight \\
\hline$A$ & $1.05 \times 10^{12} \mathrm{~cm}^{3} /(\mathrm{g} \mathrm{s})$ & Pre-Exponential Factor \\
\hline$E_{\mathrm{a}}$ & $39.2 R T_{\mathrm{o}}$ & Activation Energy \\
\hline$q$ & $59.7 R T_{\mathrm{o}} / \mathrm{M}$ & Chemical Energy Release \\
\hline$\nu_{\mathrm{O}}$ & $7.0 \times 10^{-6} \mathrm{~g} / \mathrm{s}-\mathrm{cm}-\mathrm{K}^{0.7}$ & Viscosity \\
\hline$\kappa_{\mathrm{o}}=D_{\mathrm{o}}$ & $1.0 \times 10^{-5} \mathrm{~g} / \mathrm{s}-\mathrm{cm}-\mathrm{K}^{0.7}$ & Transport Constants \\
\hline \multicolumn{3}{|c|}{ Output } \\
\hline$S_{\mathrm{L}}$ & $413 \mathrm{~cm} / \mathrm{s}$ & Laminar Flame Speed \\
\hline$T_{\mathrm{b}}$ & $11.7 T_{\mathrm{o}}$ & Post-Flame Temperature \\
\hline$\rho_{\mathrm{b}}$ & $0.085 \rho_{\mathrm{o}}$ & Post-Flame Density \\
\hline$x_{1}$ & $8.88 \times 10^{-3} \mathrm{~cm}$ & Laminar Flame Thickness \\
\hline$D_{\mathrm{CJ}}$ & $2.20 \times 10^{5} \mathrm{~cm} / \mathrm{s}$ & CJ Detonation Velocity \\
\hline$P_{\mathrm{ZND}}$ & $54.5 P_{\mathrm{o}}$ & Post-Shock Pressure \\
\hline$P_{\mathrm{CJ}}$ & $27.8 P_{\mathrm{o}}$ & Pressure at CJ Point \\
\hline$T_{\mathrm{ZND}}$ & $6 T_{\mathrm{o}}$ & Post-Shock Temperature \\
\hline$T_{\mathrm{CJ}}$ & $15.5 T_{\mathrm{o}}$ & Temperature at CJ Point \\
\hline$\rho_{\mathrm{ZND}}$ & $9.8 \rho_{\mathrm{o}}$ & Post-Shock Density \\
\hline$\rho_{\mathrm{CJ}}$ & $2 \rho_{\mathrm{o}}$ & Density at CJ Point \\
\hline$x_{\mathrm{d}}$ & $3.2 \times 10^{-4} \mathrm{~cm}$ & Half-Reaction Thickness \\
\hline$\lambda$ & $\sim 0.01 \mathrm{~cm}$ & Detonation Cell Size \\
\hline
\end{tabular}

The input parameters in Table 1 (courtesy of Alp Ozgen) were chosen to reproduce the flame and detonation properties of the ethylene-oxygen mixture initially at $1 \mathrm{~atm}$ and $298 \mathrm{~K}$. Using the input parameters in one-dimensional (1D) and two-dimensional (2D) solutions of the Navier-Stokes equations produces the output flame and detonation properties shown. Previous work [10] has shown that it is important to use a chemical-diffusive model for the conversion of reactant to product that accurately 
reproduces the major characteristics of the system. Such a model is needed because these are complex, multidimensional simulations over length scales of centimeters with reaction scales on the order of micrometers. At a basic minimum, the chemical-diffusive model must reproduce the major features of the system while still being practical computationally. The simulations in this paper used a one-step reaction model described by second-order Arrhenius kinetics (see Eqn. (7)), where the reaction rate $\dot{w}$ is proportional to $\rho$ to account for the binary nature of chemical reactions that take place in typical combustion systems. The effects of viscosity, diffusion, and heat conduction have a similar temperature dependence,

$$
\nu=\nu_{0} \frac{T^{n}}{\rho}, D=D_{0} \frac{T^{n}}{\rho}, \frac{K}{\rho C_{p}}=\kappa_{0} \frac{T^{n}}{\rho}
$$

where $\nu_{0}, D_{0}$, and $\kappa_{0}$ are constants, $C_{p}=\gamma R / M(\gamma-1)$ is the specific heat at constant pressure, and $n=0.7$ emulates the typical temperature dependence for the system. To solve Eqs. (2)-(9), we require input values for the chemical and thermophysical parameters for ethylene-oxygen that reproduce ignition, flame, and detonation properties that are similar to those computed using more detailed models or measured in experiments. The model parameters in Table 1 were determined to accurately reproduce induction delay, a 1D laminar flame with correct thickness, laminar flame velocity, adiabatic flame temperature, a 1D detonation with correct thickness, $D_{C J}$, and post-shock temperature in a stoichiometric ethylene-oxgen system. To estimate the detonation cell height, $\lambda$, for this model, a $2 \mathrm{D}$ detonation was computed on a finer mesh with 8.2 grid points per $x_{\mathrm{d}}$. Cell height ranged from 0.08 to $0.12 \mathrm{~mm}$, which is consistent with reported experimental results, which range from $0.1 \mathrm{~mm}$ to $2 \mathrm{~mm}$ $[27]$.

The computational geometry and setup is shown in Fig. 1. Obstacles are uniformly spaced along the channel wall with a constant obstacle width and height through the channel. The channel is open on the right, with symmetry planes at the top and left boundaries. The bottom wall and all obstacle surfaces are no-slip adiabatic boundaries. To ignite the mixture, a circular region of hot, burned product at $3500 \mathrm{~K}$ and $1 \mathrm{~atm}$ is placed at the left wall.

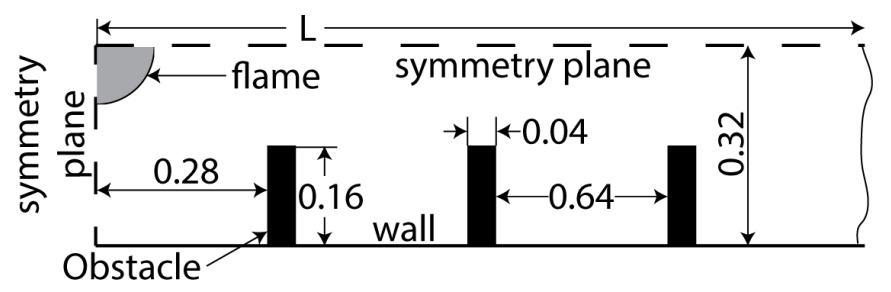

Fig. 1. Computational setup. Units of $\mathrm{cm}$. Obstacles evenly spaced along channel length $L$. Radius of the initial burned region is $0.08 \mathrm{~cm}$. Blockage ratio is 0.5 in this case. 


\section{Flame Acceleration and DDT}

\subsection{High Blockage Ratio}

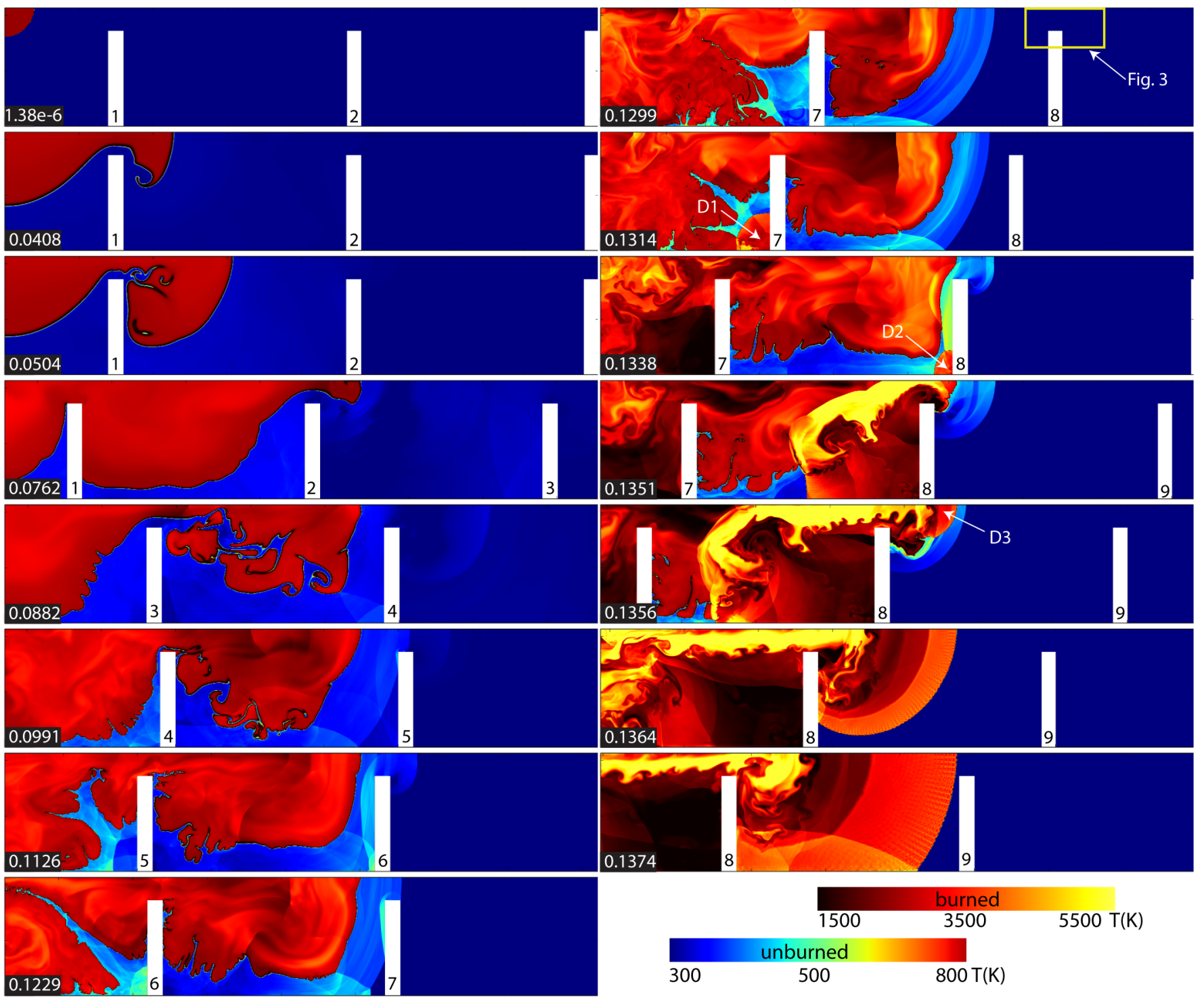

Fig. 2. Accelerating flame and detonation in $2 \mathrm{D}$ quarter-channel with $b r=0.8$. Time in milliseconds is shown in the frame corners. Obstacles are numbered. Frame width is $1.6 \mathrm{~cm}$.

In channels with high obstacles, such as the $b r=0.8$ case shown in Fig. 2, the flame accelerates rapidly as it expands over the obstacles and its surface area increases. The flame becomes increasingly turbulent due to fluid instabilities (Rayleigh-Taylor, Richtmyer-Meshkov, and Kelvin-Helmholtz). A strong shock forms immediately ahead of the flame, seen starting at $0.0991 \mathrm{~ms}$ in Fig. 2. The leading shock diffracts over obstacles, reflecting from the channel floor and forming a Mach stem behind it (0.1229 ms). As the leading shock traverses the obstacles, the strength of the Mach stem increases. The first detonation, D1, occurs in the corner in front of obstacle 7 when shocks collide and focus energy in a localized region of unburned gas $(0.1314 \mathrm{~ms})$. D1 does not survive because there is insufficient reactant available for it to traverse obstacle 7. A second detonation, D2, occurs when the leading Mach 
stem reflects from obstacle $8(0.1338 \mathrm{~ms})$. Again, there is not enough reactant available between the top of obstacle 8 and the flame for D2 to survive. The shock created by D2, however, propagates through the back of the flame into the unburned gas, causing it to detonate (D3 at 0.1356 ms). The detonation D3 survives and propagates down the channel length. Figure 3 shows the shock produced by D2 passing through the reaction front and causing the transition to detonation at $0.1353 \mathrm{~ms}$.

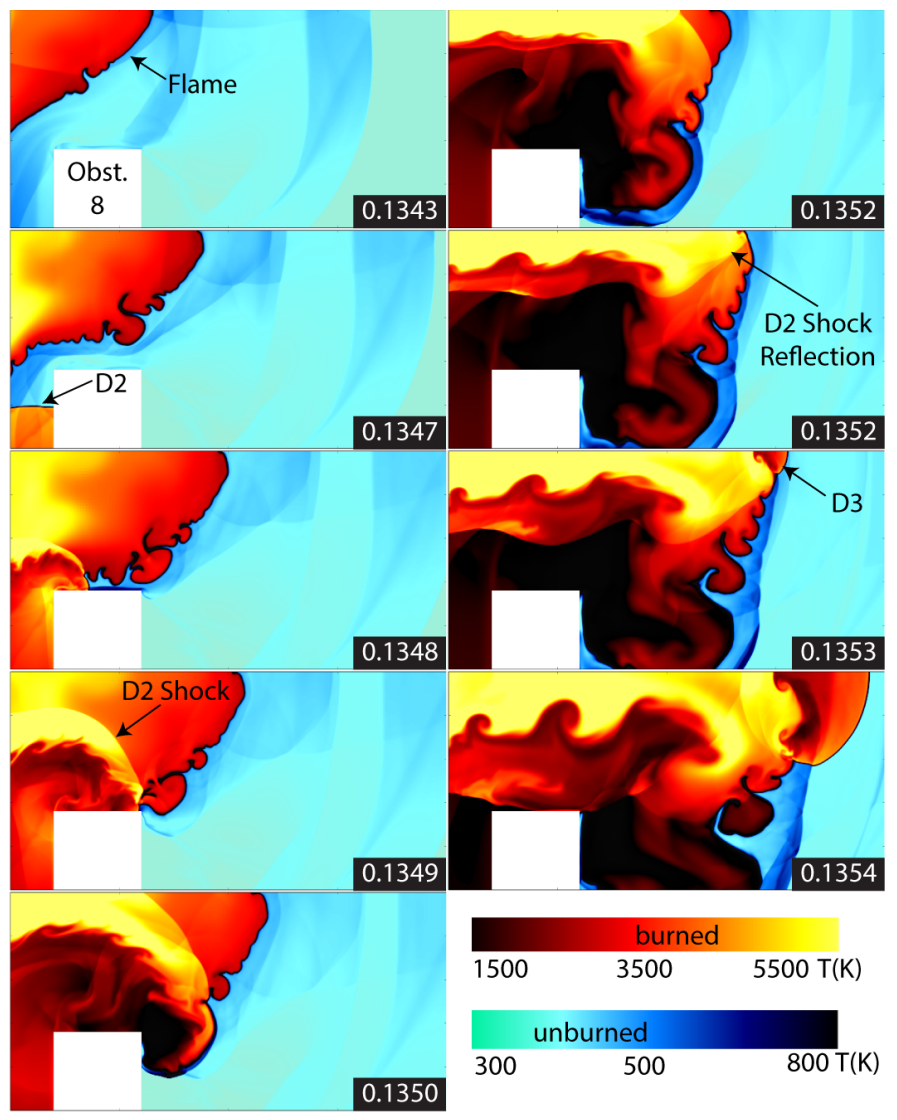

Fig. 3. Detonation with $b r=0.8$. Time in milliseconds is shown in the frame corners. Obstacle 8, detonation D2, the shock formed by D2, and D3 are labeled. Domain is $\mathrm{x}=[4.1,4.3] \mathrm{cm}$ and $\mathrm{y}$ $=[0.22,0.32] \mathrm{cm}$.

Flame acceleration and DDT in channels with $b r=0.5$ has been studied extensively for mixtures of hydrogen and air [11-13]. Figure 4 shows that the flame development is similar to that for $b r=$ 0.8. The flame accelerates due to expansion, fluid instabilities, shocks, and all of their interactions. Figure 5 shows that detonation occurs in preheated, unburned gas as a Mach stem reflects from an obstacle and leaves a hot spot in a gradient of reactivity. Pressure and temperature increase behind the Mach reflection, igniting two hot spots at $0.1356 \mathrm{~ms}$. The hot spots become detonations through the gradient mechanism. Detonation occurs $3.5 \mathrm{~cm}$ from the left edge after the flame has passed six obstacles. 


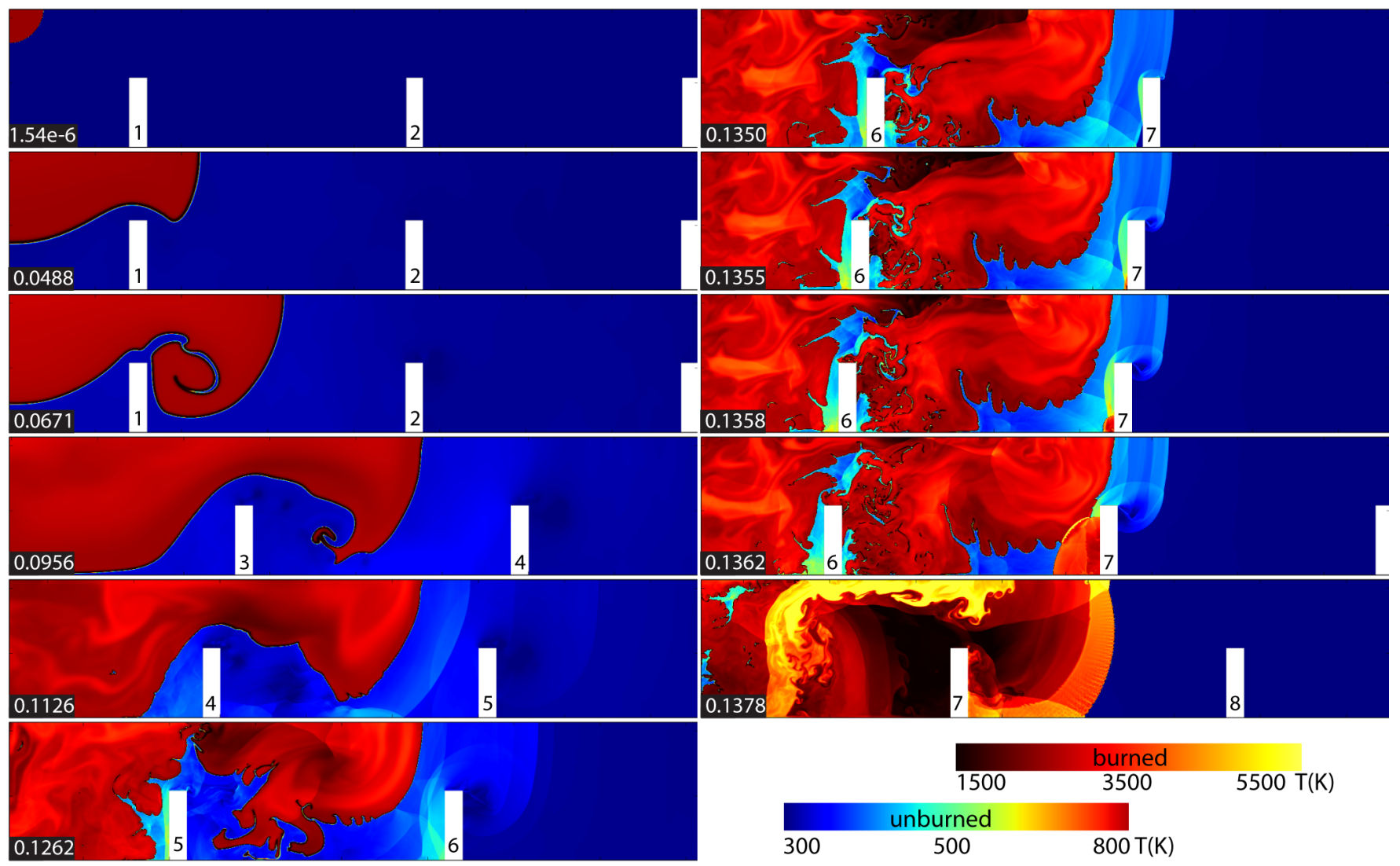

Fig. 4. Accelerating flame and detonation in $2 \mathrm{D}$ quarter-channel with $b r=0.5$. Time in milliseconds is shown in the frame corners. Obstacles are numbered. Frame width is $1.6 \mathrm{~cm}$.

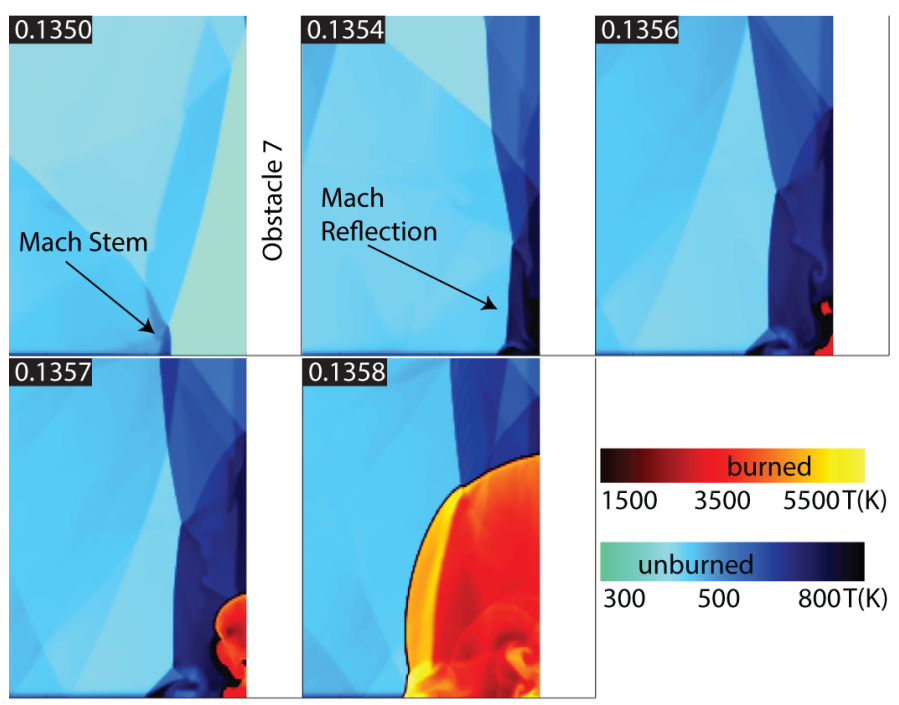

Fig. 5. Detonation with $b r=0.5$. Time in milliseconds is shown in the frame corners. Obstacle 7 shown on right side. Domain is $\mathrm{x}=[3.4,3.5] \mathrm{cm}$ and $\mathrm{y}=[0,0.1] \mathrm{cm}$.

\subsection{Intermediate Blockage Ratio}

When $b r=0.1$, detonation occurs at $7.25 \mathrm{~cm}$ against the lower wall at $0.271 \mathrm{~ms}$, after the flame has passed 11 obstacles. Unburned gas is pushed downstream over obstacles causing vortex shedding from the obstacle's leading edges (seen starting at $0.1804 \mathrm{~ms}$ in Fig. 6). Vortices coalesce behind obstacles to form recirculation regions. The flame surface increases as it is pulled into the recirculation region, 
and the flame becomes turbulent as the pressure waves created by the compressible vortices propagate through the flame. Shocks form as the flame accelerates, and these shocks increase in strength as they reflect off the obstacles and the channel boundaries. Fluid instability in the unburned region behind the leading shock increases as the shocks collide and interact with boundary layers and vortices.

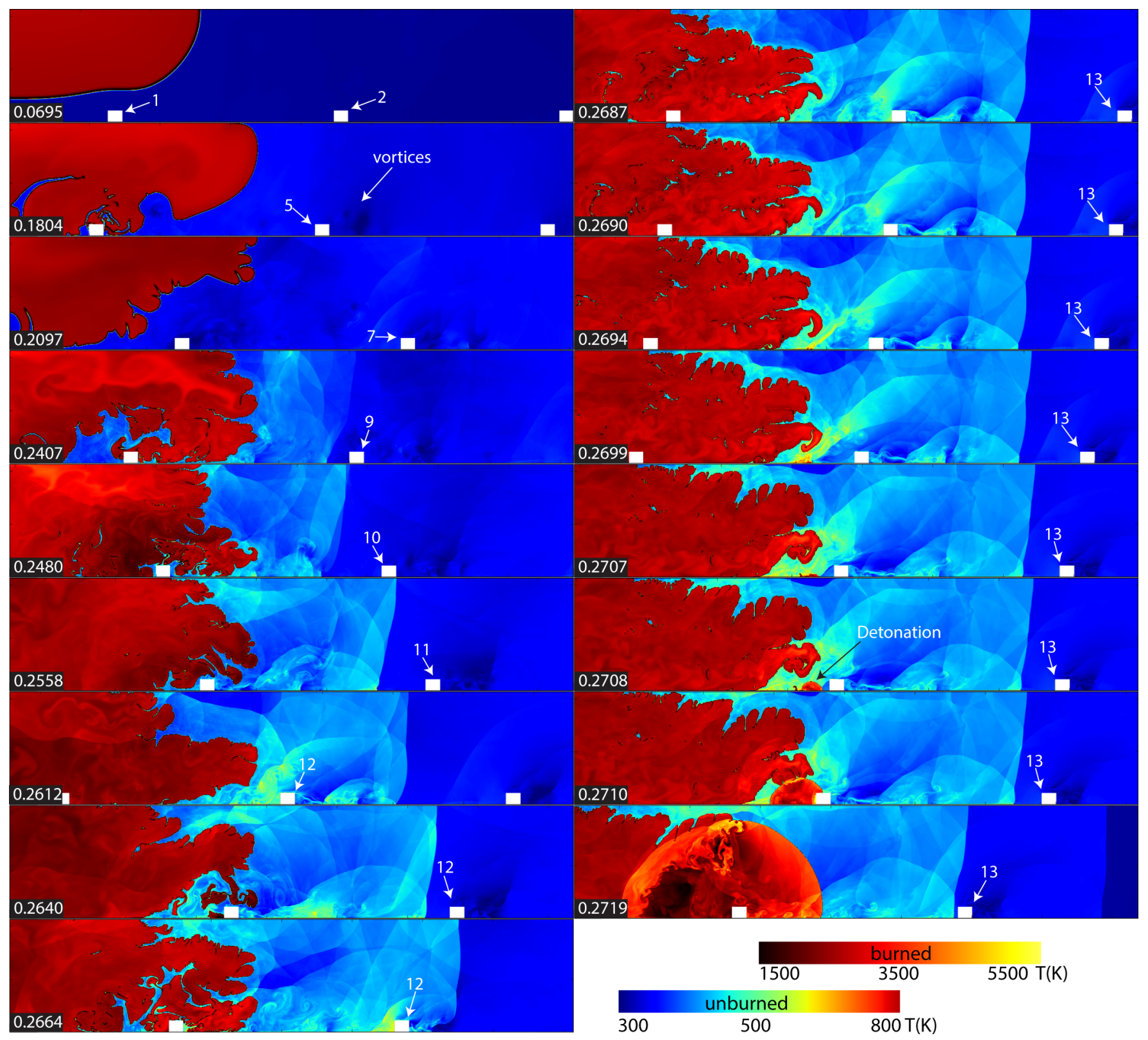

Fig. 6. Full simulation for blockage ratio of 0.10. Time in milliseconds is shown in the frame corners. Obstacles are numbered. Frame width is $1.6 \mathrm{~cm}$.

Figure 7 is an enlargement of the detonation that occurs in Fig. 6 for $b r=0.1$. Two shocks, labeled $\mathrm{S} 1$ and S2 at $0.2689 \mathrm{~ms}$, collide at $0.2696 \mathrm{~ms}$. Shock S1 is immediately ahead of the flame, traveling to the right, and shock S2 is reflected from the downstream obstacle (shown in white in the lower right corner of each frame in Fig. 7) and travels to the left. A shock collision ignites two hot spots in the boundary layer, labeled H1 and H2. By 0.2707 ms, H1 evolves into a flame and shock, and H2 becomes a detonation. The analysis in Section 5.2 shows that the confluence of shocks causes transition to 
detonation in hot unburned gas in this intermdediate $b r$ regime through a detonation mechanism that has elements of both gradient ignition and direct initiation of detonation. This is also discussed in [22].

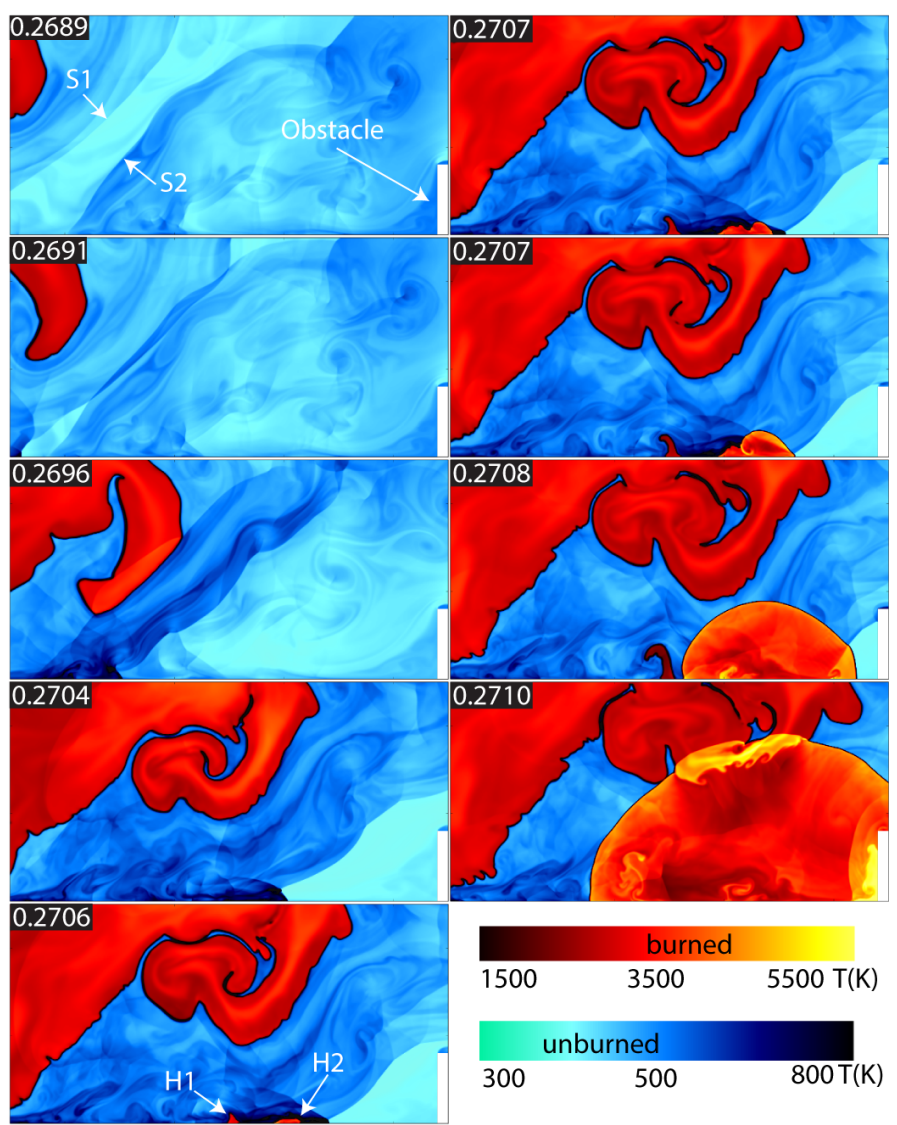

Fig. 7. Multiple shock collision and detonation for $b r=0.1$. Time in milliseconds is shown in the frame corners. Domain is $\mathrm{x}=[7.125,7.325] \mathrm{cm}$ and $\mathrm{y}=[0,0.1] \mathrm{cm}$.

\subsection{Low Blockage Ratio}

In the low $b r$ regime, the flame accelerates very slowly. A relatively large, unburned, turbulent region filled with reflecting shocks and strong vortices forms between the flame and the leading shock. Figure 8 shows the complexity and dynamic structure in this shock-flame complex prior to DDT. Detonation, shown in Fig. 9, occurs at $0.5562 \mathrm{~ms}$ at $16.8 \mathrm{~cm}$, after the flame passed 27 obstacles. A shock ahead of the flame, labeled S3, reflects against obstacle 28 and collides with shock S4, increasing the pressure and temperature of the gas subjected to the double shock collision. S4 then forms a Mach stem against the channel wall. A hot spot and subsequent detonation form when the Mach stem collides with obstacle 28.

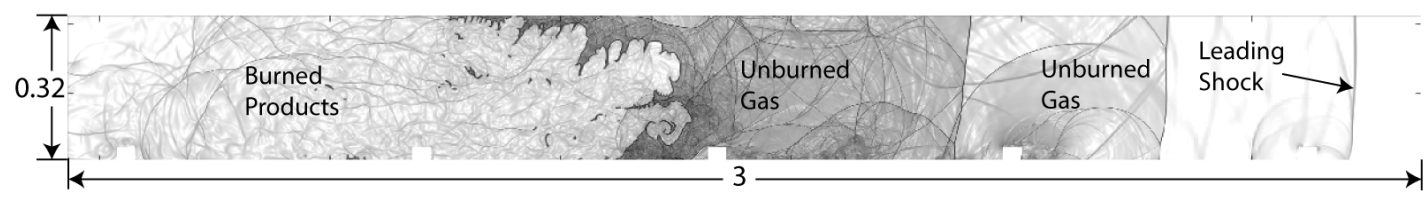

Fig. 8. Numerical schlieren, $|\nabla \rho|$, showing the turbulent region for $b r=0.05$. Dimensions are in $\mathrm{cm}$. 


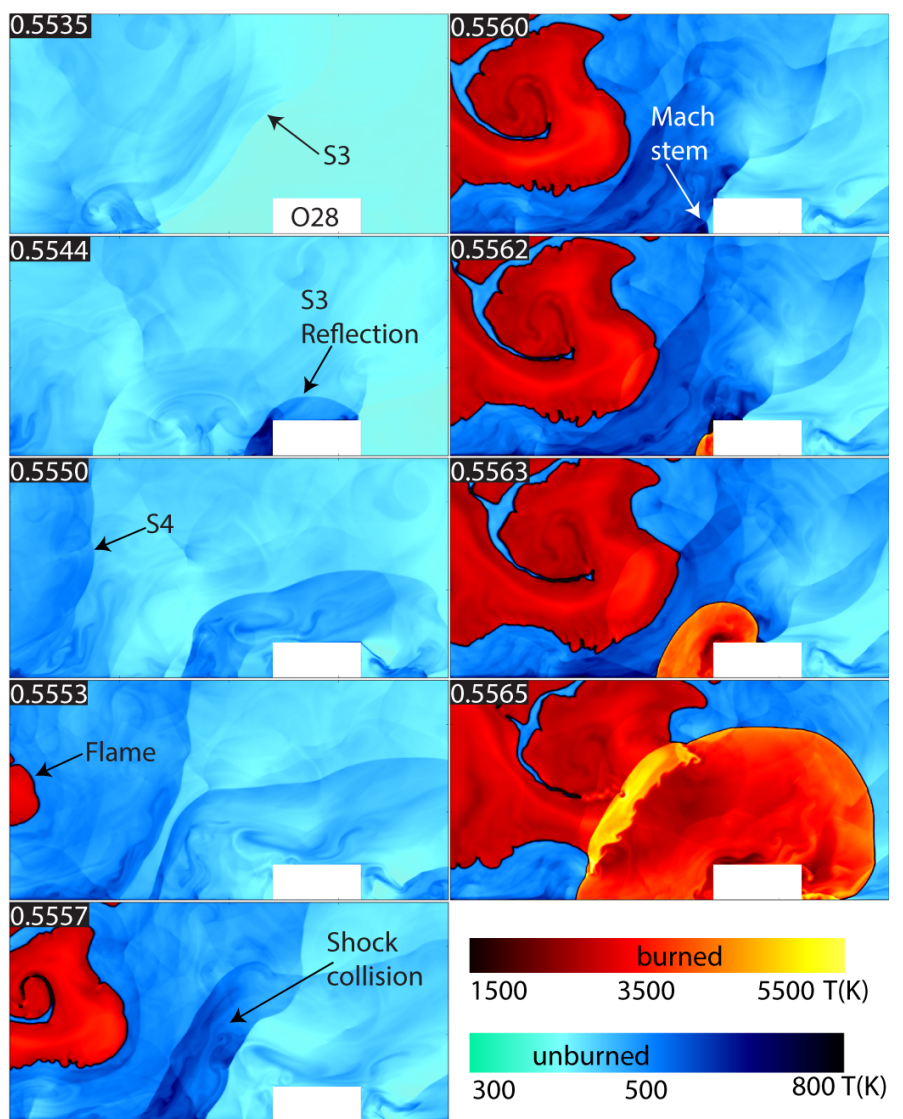

Fig. 9. Detonation with $b r=0.05$. Time in milliseconds is shown in the frame corners. Obstacle 28 is labeled. Domain is $\mathrm{x}=[16.8,17.0] \mathrm{cm}$ and $\mathrm{y}=[0,0.1] \mathrm{cm}$.

\subsection{Comparison of DDT in Two and Three Dimensions}

Another set of simulations with a channel height of $0.16 \mathrm{~cm}$ and $b r=0.1$ was performed to compare 2D and 3D flame acceleration and DDT. The channel height was decreased from $0.32 \mathrm{~cm}$ to $0.16 \mathrm{~cm}$ to reduce computational expense in the 3D calculation. Figure 10 shows the computational setup, now for which $d x_{\min }=6.67 \mu m$ and $d x_{\max }=53.33 \mu \mathrm{m}$. Despite the difference in the two channel heights, the DDT mechanism is the same. The detonation in the 2D simulation with a reduced channel height is shown in Fig. 11. Detonation occurs after the flame has passed 13 obstacles at $0.1472 \mathrm{~ms}, 4.11 \mathrm{~cm}$ into the channel. The accelerating flame creates pressures waves that coalesce into a shock and the shock reflects from the channel wall. The shock reflection passes through the turbulent flame front, causing the flame front to transition to a detonation, as it does in the case with $b r=0.8$ (see D3 in Fig. 3).

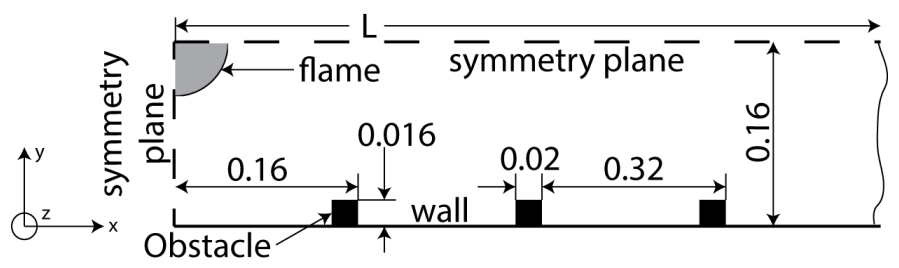

Fig. 10. Computational setup for 3D calculation in units of $\mathrm{cm}$. Obstacles evenly spaced along channel length $L$. Radius of the initial burned region is $0.04 \mathrm{~cm}$. 


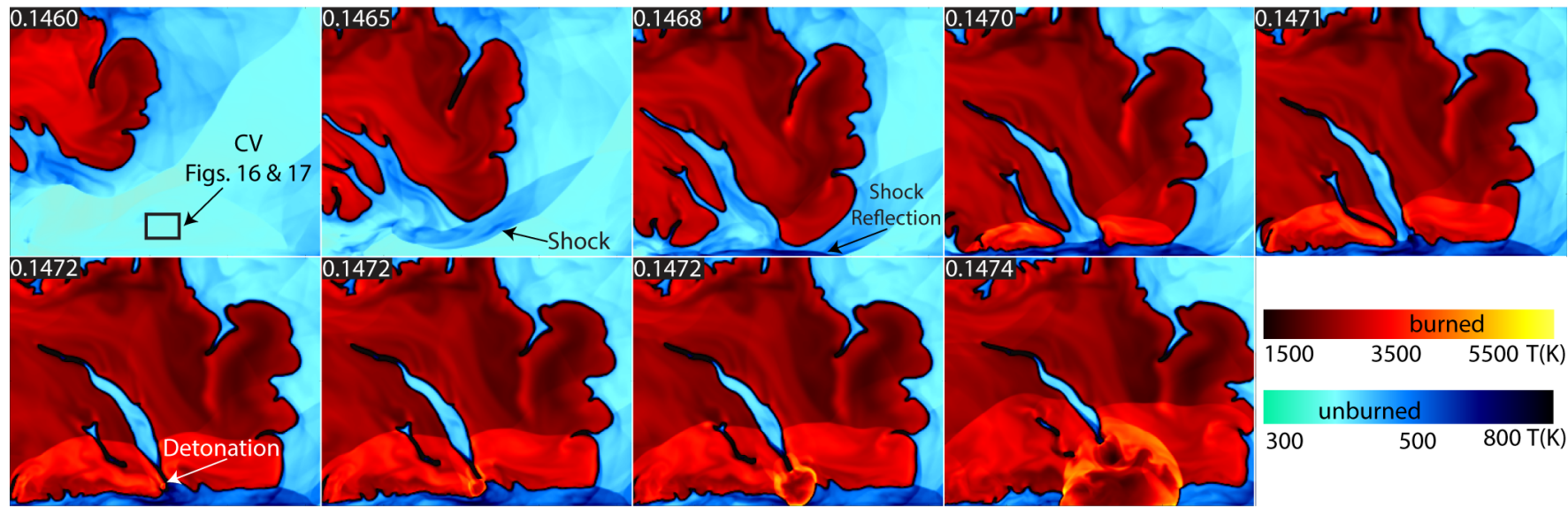

Fig. 11. Detonation with $b r=0.1$ and a channel height of $0.16 \mathrm{~cm}$. Time in milliseconds is shown in the frame corners. Domain is $\mathrm{x}=[4.01,4.21] \mathrm{cm}$ and $\mathrm{y}=[0,0.16] \mathrm{cm}$.

In the $3 \mathrm{D}$ case, the channel width is $0.16 \mathrm{~cm}$ with periodic boundary conditions in the $\mathrm{z}$ direction, with coordinate axes shown in Fig. 10. The turbulent flame acceleration and transition to detonation is shown in Fig. 12. The evolution of the flame and eventual DDT is similar to what is observed in a 2D channel with intermediate $b r$. The initially laminar flame accelerates as it traverses the obstacles. A turbulent flame structure forms near the bottom wall after the flame has passed three obstacles, shown at $0.071 \mathrm{~ms}$. After passing six obstacles, the entire flame front has become turbulent. At 0.102 ms, the unburned mixture detonates near the bottom wall, $3.05 \mathrm{~cm}$ down the length of the channel, due to the same mechanism of energy deposition we see in the $2 \mathrm{D}$ simulations for intermediate $b r$. The detonation begins near the center of the channel width and propagates down the length of the channel (x direction), forming cell structure as shown at $0.113 \mathrm{~ms}$. The multidimensional turbulence in the $3 \mathrm{D}$ case leads to a faster rate of flame acceleration and earlier detonation than in the $2 \mathrm{D}$ case. Figure 13 shows the location of the tip of the reaction front as a function of time for the $2 \mathrm{D}$ and $3 \mathrm{D}$ cases.

\section{Discussion}

\subsection{Effect of Blockage Ratio}

A comparison of the simulations with high and low $b r$ shows that obstacle size plays a major role in flame acceleration, turbulence in the flame and unburned gas, shock formation, time or distance to detonation, and thus in the detonation mechanism. Figure 14 shows the position of the tip of the reaction front as a function of time for all blockage ratios studied. In the regime of high $b r$, the time to detonation increases from $0.136 \mathrm{~ms}$ for $b r=0.8$ to $0.161 \mathrm{~ms}$ for $b r=0.35$. In the low $b r$ regime, it takes considerably longer for DDT to occur, here at $0.556 \mathrm{~ms}$ for $b r=0.05$. Figure 15 shows the number of obstacles traversed by the tip of the reaction front prior to DDT for each $b r$. Higher obstacles cause the flow to become turbulent faster, as the higher obstacles perturb the flame more than smaller obstacles. 

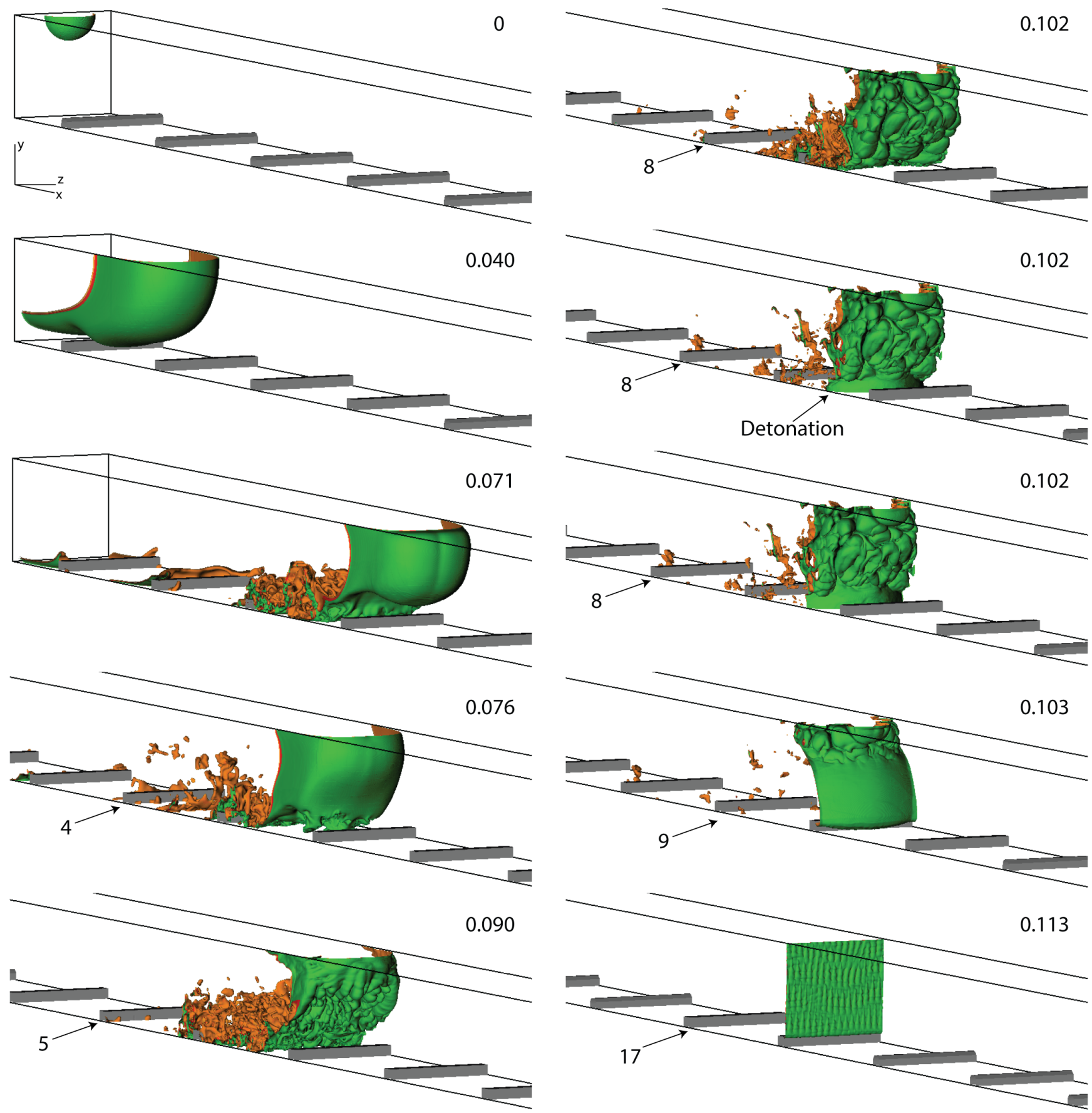

Fig. 12. Isosurfaces of reactant mass fractions, $Y$, of $0.2,0.5$, and 0.8 show flame acceleration and detonation in 3D channel with $b r=0.1$. Time in milliseconds is shown in the frame corners. Obstacles are numbered.

A reverse relationship holds for the $b r=0.8$ case, where the reaction front traverses 7 obstacles before DDT, as opposed to the 6 obstacles before DDT in the $b r=0.5$ and 0.35 cases. The reaction front passes more obstacles before DDT when the $b r=0.8$ because the obstacles in this case are high enough to choke the initial laminar expansion of the flame, resulting in slower initial acceleration than in the $b r=0.5$ and 0.35 cases. In the $b r=0.05$ case, the obstacles take much longer to perturb the flame and create shocks of sufficient strength to detonate the unburned gas. When $b r=0.05$, the reaction front 


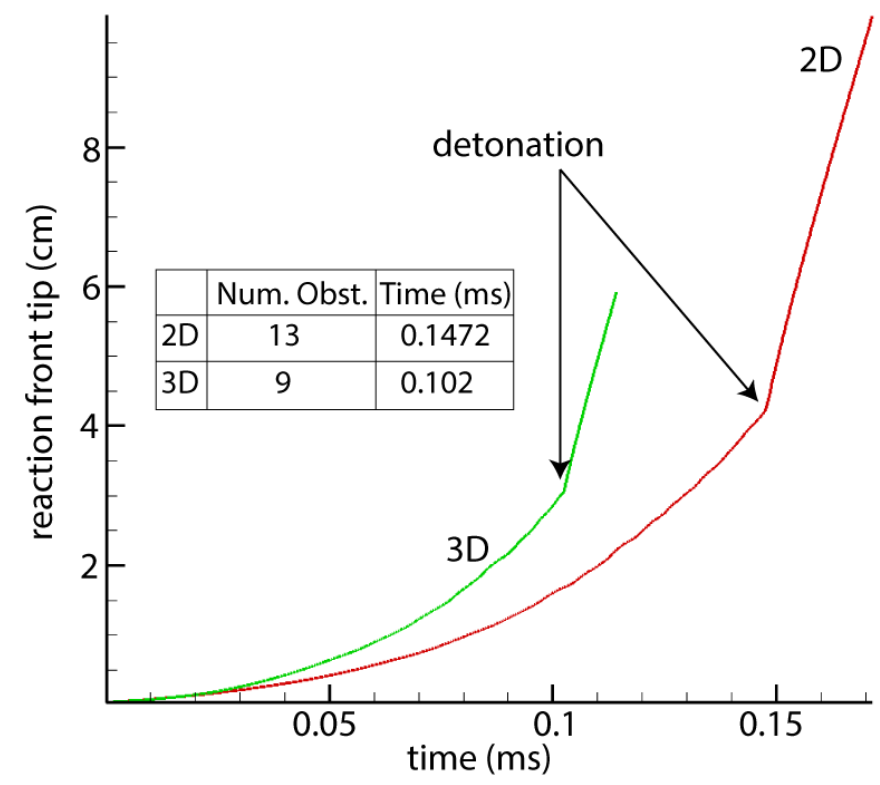

Fig. 13. Reaction front tip position vs. time for $2 \mathrm{D}$ and $3 \mathrm{D}$ simulations with a channel height of 0.16 $\mathrm{cm}$ and $b r=0.1$. The time and number of obstacles to detonation is shown.

traverses 28 obstacles prior to detonation.

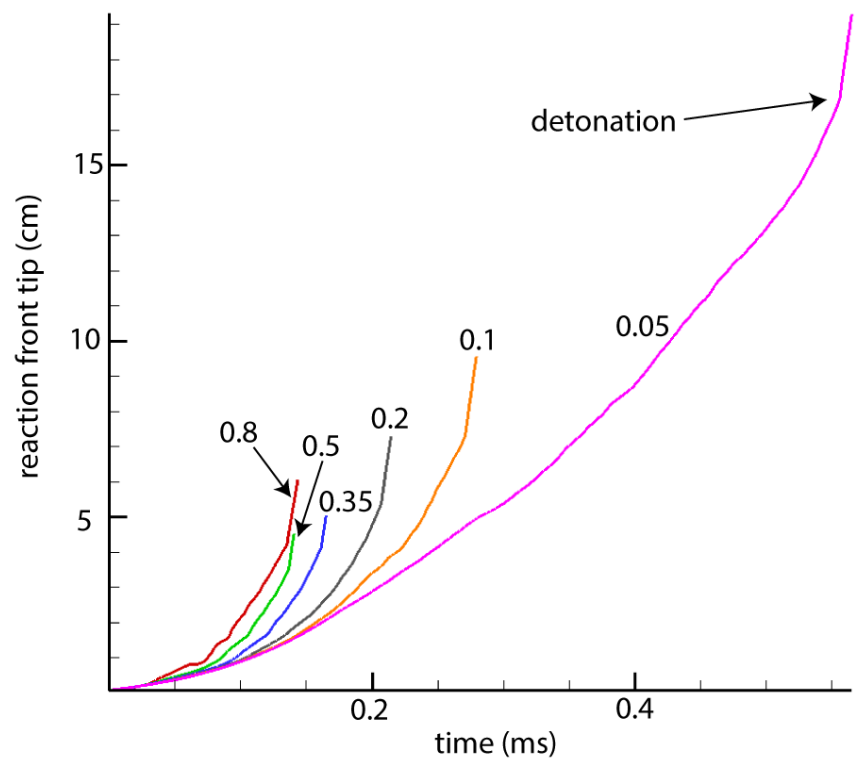

Fig. 14. Reaction front tip as a function of time for all blockage ratios in this series of simulations. Detonation occurs at the inflection point, as shown for $b r=0.05$.

When $b r$ is in the intermediate range $(b r=0.1$ in Fig. 6), the flame accelerates more slowly and stays laminar further into the channel than it does for higher obstacles ( $\operatorname{such}$ as $b r=0.5$, in Fig. 4). Higher values of $b r$ result in a more predictable flow as strong leading shocks form very close to the flame. These obstacles consistently produce Mach-stems and detonations that form behind Mach-stem reflections. For this stoichiometric ethylene-oxygen mixture, the flame never falls more than $\sim 0.2 \mathrm{~cm}$ behind the leading shock for $b r=0.5$. When $b r$ decreases to 0.1 , the leading shocks form as far as 1.5 cm ahead of the flame tip, creating a large, unburned turbulent region ahead of the flame. Rather than 


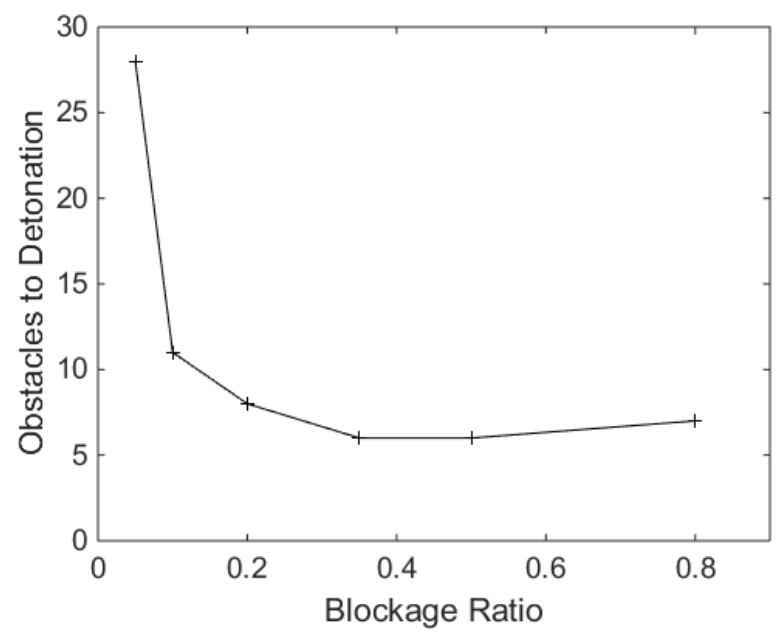

Fig. 15. Number of obstacles to detonation as a function of blockage ratio

a detonation always occurring in a Mach reflection against an obstacle, it may occur anywhere that energy is focused sufficiently to initiate the detonation. The actual detonation location is stochastic because the turbulent region is filled with shock collisions and reflections that may focus sufficient energy to detonate the unburned gas anywhere within the unburned, turbulent region ahead of the flame.

The case with $b r=0.8$ is particularly interesting because both DDT mechanisms arise. As shown in Fig. 2, detonation D2 occurs in a gradient of reactivity behind a Mach-stem reflection. Due to the height of the obstacles, there is insufficient reactant available between the burned gas and the obstacle for the D2 detonation front to traverse the obstacle and propagate through the channel. When the shock produced by D2 passes through the burned gas and the reaction front, it causes DDT through the SWACER mechanism. The shock couples with the flame and injects sufficient energy into the chemically reacting gas to cause the flame to transition to detonation D3. This process of shocks causing a flame front to transition to detonation is also seen in the $2 \mathrm{D}, 0.16 \mathrm{~cm}$ channel height case, as shown in Fig. 11.

\subsection{Shock Focusing and Rapid Energy Deposition}

A control volume analysis was performed on a small, $33.3 \mu \mathrm{m} \times 33.3 \mu \mathrm{m}$ region (marked in the first frame of Fig. 11) where the detonation emerges from the flame front at $0.1472 \mathrm{~ms}$. The analysis tracked the change in pressure, temperature, density, and energy-release rate over a $0.45 \mu$ s period from 146.9 $\mu \mathrm{s}$ to $147.35 \mu \mathrm{s}$. This covers the time before the region is compressed by the shock reflection to a time after the detonation formed and propagated through the region. Figure 16 shows the trends of the state variables during this time period. Shock compression begins at $146.9 \mu \mathrm{s}$, and the material begins to react chemically at $147.15 \mu \mathrm{s}$. As the material is compressed by the shock reflection, the pressure and 
density increase dramatically. There is another spike when the mixture detonates. As the detonation propagates out of the region, the pressure and density decrease.

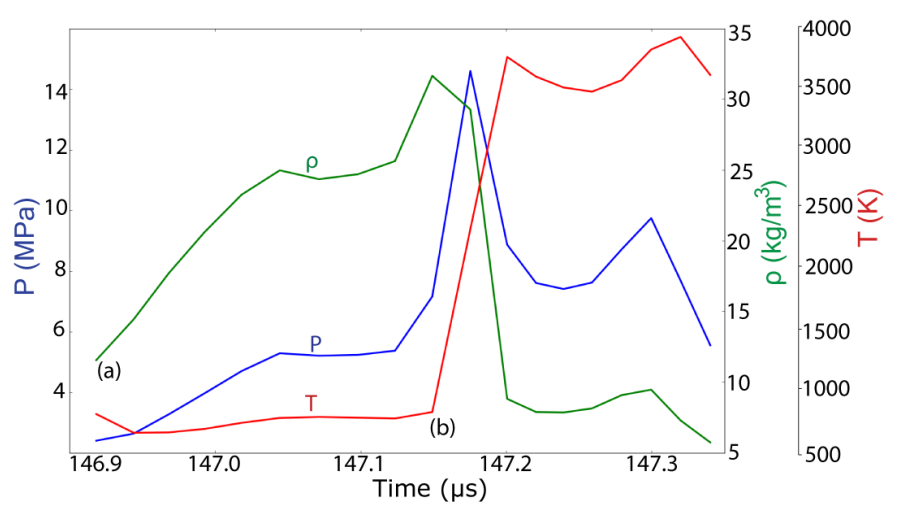

Fig. 16. State variables in the control volume from $146.9 \mu$ s to $147.35 \mu$ s. Shock compression begins at (a). Detonation occurs at (b). The gas begins to react chemically at $147.15 \mu \mathrm{s}$.

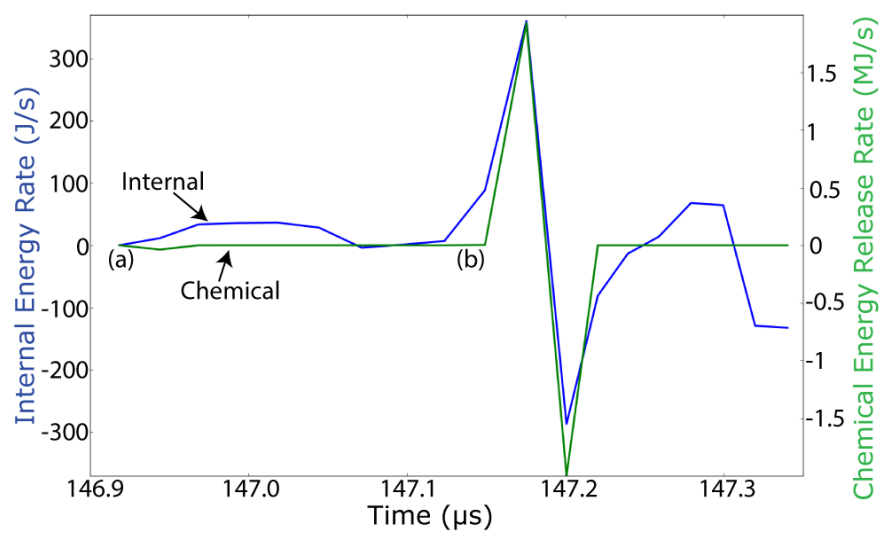

Fig. 17. Rate of energy deposition in control volume. Shock compression begins at (a). Detonation occurs at (b).

Figure 17 shows the rate of energy deposition into the control volume. Chemical energy release is defined as heat release per $\mathrm{kg}$ of reactant, $q$, and sensible internal energy is $P /(\gamma-1)$. As the material in the region is compressed, the density, pressure, and internal energy increase. There is a second peak as the material detonates and chemical energy is released. The flow behind the detonation wave carries energy out of the region as the detonation propagates into the surrounding gas, resulting in the negative energy rates seen after $147.17 \mu \mathrm{s}$. The total energy content in the region, defined as the sum of chemical, internal, and kinetic energies, increases by a factor of 16 during shock compression. A similar analysis was performed in earlier work [22] for the case shown in Fig. 6 with $b r=0.1$ and a channel height of $0.32 \mathrm{~cm}$. The analysis tracked the rate of energy deposition into a region of unburned gas subjected to multiple shock collisions resulting in a detonation, as shown in Fig. 7. The trends shown here in Figs. 16 and 17 are consistent with the earlier analysis. The shock compression causes a rapid increase in pressure, temperature, and density. The result is an increase in the rate of energy deposition, followed by direct initiation of a detonation in the unburned gas. 


\subsection{Stochastic Analysis}

Prior work [11] has shown that for hydrogen and air mixtures in channels of different sizes, there were different regimes of DDT. The authors found that in some of these regimes, the detonation mechanism was more sensitive to the turbulence in the flow. The variations in $b r$ described in this paper reflect the same phenomenon, that is, that there are different regimes of DDT with varying sensitivity of the detonation mechanism to turbulence. In the $b r=0.1$ intermediate regime, the sensitivity of the solution to turbulence causes the detonation to occur at slightly different times and locations for cases with modified initial conditions. Following [11], we have now shown that simulations for cases in which the initial background temperature is varied within $298 \pm 0.01 \mathrm{~K}$, the location and time of detonation change slightly, but the mechanism remains the same. Figure 18 shows the reaction-front tip position as a function of time for simulations with $b r=0.1$ and for slightly varied initial background temperature. The time to detonation differs within a range of $0.05 \mathrm{~ms}$ and the distance to detonation varies within a $2.4 \mathrm{~cm}$ range.

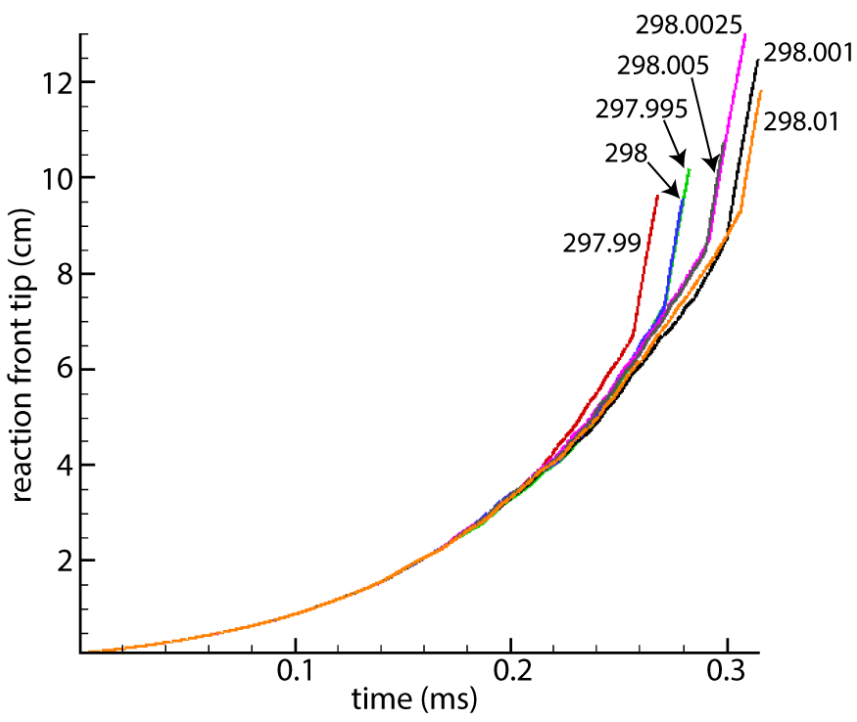

Fig. 18. Position of the reaction-front tip as a function of time for $b r=0.1$ for background temperatures (labeled in $\mathrm{K}$ ) in the range $298 \pm 0.01 \mathrm{~K}$. Range in time and distance to detonation are $0.05 \mathrm{~ms}$ and 2.4 $\mathrm{cm}$, respectively.

Stochasticity in cases with $b r=0.1$ can also be seen when the grid size is varied. Figure 19 shows the detonation in another simulation with $b r=0.1$ with the same minimum grid size as in the original $b r=0.1$ case shown in Fig. 6 , but with a finer coarse grid $(26.7 \mu \mathrm{m}$ instead of $53.3 \mu \mathrm{m})$. The location of the detonation is $\sim 1.2 \mathrm{~cm}$ further along the channel boundary than the detonation that occurred in the original $b r=0.1$ case (shown in Fig. 7). Nevertheless, a detonation again appears where shock collisions and reflections are focused. The shock immediately ahead of the flame, labeled S5, reflects off the channel boundary and ignites a hot spot H3 in the boundary layer. The hot spot becomes a detonation while another shock ahead of the flame, labeled S6, reflects off the obstacle shown in the 
lower right corner of the figure. The reflection of S6 ignites another hot spot, labeled H4, that also becomes a detonation. The two detonations combine and propagate down the channel. Turbulence, an essentially stochastic process, dominates this region of fluid instabilities, shock collisions, and shockboundary layer interactions.

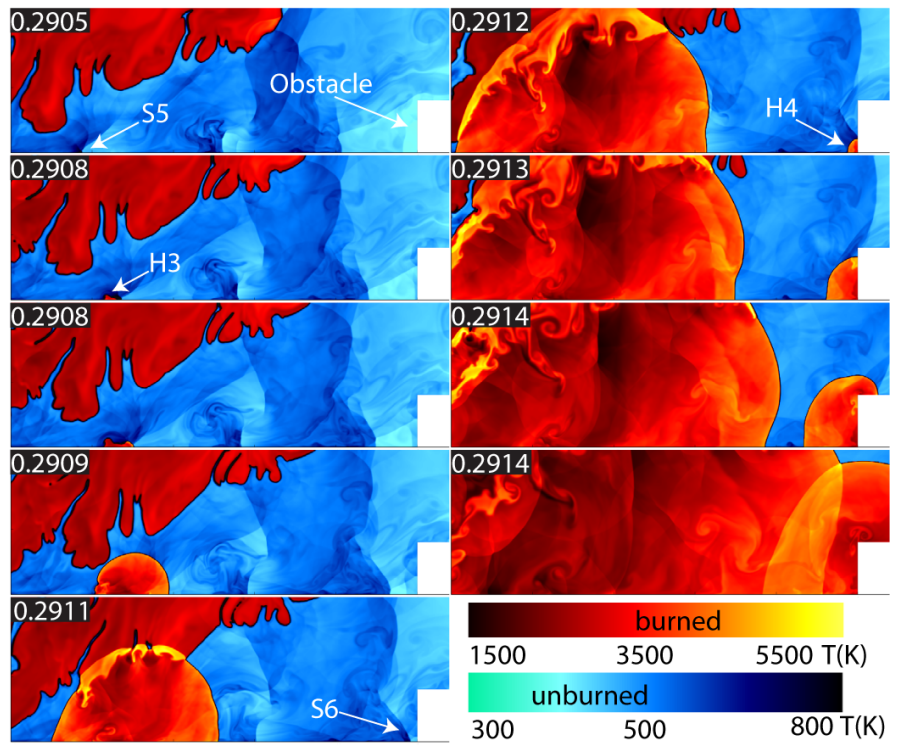

Fig. 19. Shock reflections and collisions result in initiation of two detonations. Time in milliseconds is shown in the frame corners. Domain is $\mathrm{x}=[8.35,8.65] \mathrm{cm}$ and $\mathrm{y}=[0,0.2] \mathrm{cm}$.

\section{Summary and Conclusions}

The effect of the blockage ratio $(b r)$ on the mechanism of DDT was studied by analyzing a series of multidimensional, unsteady numerical simulations of an initially homogeneous mixture of ethylene and oxygen in partially blocked channels. This paper compared several cases taken from a series of simulations with $b r=0.05-0.8$, including a three-dimensional (3D) calculation.

For $b r=0.35-0.5$, detonation occurs due to the creation of a hot spot in a gradient of reactivity that forms behind a Mach reflection [11-13]. This was shown for different fuels than were used in prior work. In the case with $b r=0.05-0.2$, shock collisions increase the temperature, pressure, and density of the unburned material. These effects and interactions occur frequently due to the complexity of the shock structures and interactions in the unburned turbulent region between the flame and the leading shock. As the flame propagates down the channel, the strength of the shock collisions and reflections increases in this region. Multiple shocks collide and reflect against the channel boundary, focusing energy in a localized volume of unburned material and detonating the mixture. These results are consistent with direct initiation theory [16] and with detonations seen in experiments using similar blockage ratios $(b r<0.3)[23]$. Sufficient energy is deposited into a localized region on a timescale that is small relative to the acoustic timescale of the gas, thus initiating a detonation. DDT by rapid energy 
deposition into a localized region at a very small timescale was also seen in a 3D calculation with $b r$ $=0.1$, validating two-dimensional $(2 \mathrm{D})$ results. Increased turbulence in the $3 \mathrm{D}$ calculation led to a faster flame acceleration and earlier DDT than in the comparable 2D case, although the detonation mechanism remained the same.

The case of high blockage ratio, $b r=0.8$, showed both mechanisms. A hot spot ignited in a gradient of reactivity formed behind a Mach reflection, but the detonation failed due to insufficient reactant for the detonation front to traverse the upstream obstacles. The shock created by the second detonation propagated through the burned products, through the reaction front, and into the unburned gas. As the shock passed through the reaction front, the flame transitioned to detonation. The detonation mechanism here was shock focusing, as the reaction front was compressed by the shock and enough energy was deposited for transition to detonation. This same phenomenon is seen in the $2 \mathrm{D}, 0.16 \mathrm{~cm}$ channel height simulation with $b r=0.1$.

An additional series of simulations, with $b r=0.1$, investigated the sensitivity of the solution to initial conditions. Modifying the initial background temperature, within the range of $298 \pm 0.01 \mathrm{~K}$ resulted in a difference in detonation time and location, but the detonation mechanism was the same. This phenomenon was also seen in previous work with a different fuel mixture [11], where it was determined that some regimes of DDT are especially sensitive to turbulence in the flow.

\section{Acknowledgments}

This work was supported by the Office of Naval Research, the University of Maryland through Minta Martin Endowment Funds in the Department of Aerospace Engineering, and the Glenn L. Martin Institute Chaired Professorship at the A. James Clark School of Engineering. The computations were carried out on University of Maryland supercomputing resources (http://www.it.umd.edu/hpcc). The authors thank David Kessler and Fokion Egolfopoulos for their assistance in developing the chemical model used in these simulations, as well as Vadim Gamezo for sharing his insights on DDT in channels with obstacles. The authors particularly acknowledge the help of Alp Ozgen in determining the optimal chemical-diffusive model.

\section{References}

[1] P. Middha, O. Hansen, Predicting deflagration to detonation transitions in hydrogen explosions, Process Saf. Prog. 27 (2008) 192-204.

[2] P. Thibault, L. Britton, F. Zhang, Deflagration and detonation of ethylene oxide vapor in pipelines, Process Saf. Prog. 19 (2000) 125-139.

[3] R. Sorin, R. Zitoun, D. Desbordes, Optimization of the deflagration to detonation transition: reduction of length and time of transition, Shock Waves 15 (2006) 137-145. 
[4] R. Smirnov, R. Zitoun, D. Desbordes, Deflagration-to-detonation transition in gases in tubes with cavities, J. Eng. Phys. Thermophys. 83 (2010) 1287-1316.

[5] Y. Huang, H. Tang, J. Li, J. Wang, Deflagration-to-detonation transition of keroseneair mixtures in a small-scale pulse detonation engine, J. Aerosp. Eng. 225 (2011) 441-448.

[6] R. Zipf, V. , M. Sapko, W. Marchewka, K. Mohamed, E. Oran, D. Kessler, E. Weiss, J. Addis, F. Karnack, D. Sellers, Methane-air detonation experiments at niosh lake lynn laboratory, J. Loss Prevention Process Indus. 26 (2013) 295-301.

[7] J. Lee, A. Higgins, Comments on criteria for direct initiation of detonation, Phil. Trans. R. Soc. Lond. 357 (1999) 3503-3521.

[8] B. Zhang, H. Ng, J. Lee, Measurement and relationship between critical tube diameter and critical energy for direct blast initiation of gaseous detonations, J. Loss Prevention Process Indus. 26 (2013) $1293-1299$.

[9] J. Lee, The Detonation Phenomenon, 1 ed., Cambridge University Press, New York, NY, 2008.

[10] E. S. Oran, V. N. Gamezo, Origins of the deflagration-to-detonation transition in gas-phase combustion, Combust. Flame 148 (2007) 4-47.

[11] V. N. Gamezo, T. Ogawa, E. S. Oran, Flame acceleration and ddt in channels with obstacles: Effect of obstacle spacing, Combust. Flame 155 (2008) 302-315.

[12] V. N. Gamezo, T. Ogawa, E. S. Oran, Numerical simulations of flame propagation and ddt in obstructed channels filled with hydrogen-air mixture, Proc. Combust. Inst. 31 (2007) 2463-2471.

[13] D. A. Kessler, V. N. Gamezo, E. S. Oran, Simulations of flame acceleration and deflagration-todetonation transitions in methane-air systems, Combust. Flame 157 (2010) 2063-2077.

[14] G. Ciccarelli, C. Fowler, M. Bardon, Effect of obstacle size and spacing on the initial stage of flame acceleration in a rough tube, Shock Waves 14 (2005) 161-166.

[15] A. Na'Inna, H. Phylaktou, G. Andrews, Effects of obstacle separation distance on gas explosions: the influence of obstacle blockage ratio, Procedia Eng. 84 (2014) 306-319.

[16] A. D. Kiverin, D. R. Kassoy, M. F. Ivanov, M. A. Liberman, Mechanisms of ignition by transient energy deposition: Regimes of combustion wave propagation, Phys. Rev. E 87 (2013) 033015.

[17] D. Kassoy, The response of a compressible gas to extremely rapid transient, spatially resolved energy addition: an asymptotic formulation, J. Eng. Math. 68 (2010) 249-262.

[18] Y. Zeldovich, V. Librovich, G. Makhviladze, G. Sivashinsky, On the development of detonation in a non-uniformly preheated gas, Astronaut. Acta 15 (1970) 313-321.

[19] J. Lee, R. Knystautas, N. Yoshikawa, Photochemical initiation of gaseous detonations, Acta Astronaut. 5 (1978) 971-982.

[20] G. Ciccarelli, S. Dorofeev, Flame acceleration and transition to detonation in ducts, Prog. Energy Combust. Sci. 34 (2008) $499-550$.

[21] V. N. Gamezo, T. Ogawa, E. S. Oran, Deflagration-to-detonation transition in h2-air mixtures: Effect of blockage ratio, AIAA Paper 440 (2009).

[22] G. Goodwin, R. Houim, O. Oran, Shock transition to detonation in channels with obstacles, 36th International Symposium on Combustion (2016).

[23] S. Maeda, S. Minami, D. Okamoto, T. Obara, Visualization of deflagration-to-detonation transitions in a channel with repeated obstacles, in: 25th International Colloquium on the Dynamics of Explosions and Reactive Systems, 2015. 
[24] R. W. Houim, K. K. Kuo, A low-dissipation and time-accurate method for compressible multicomponent flow with variable specific heat ratios, J. Comput. Phys. 230 (2011) 8527-8553.

[25] Center for Computational Sciences and Engineering, University of California, Berkeley, Boxlib, Retrieved 03-2015. URL: https://ccse.lbl.gov/index.html.

[26] A. Chaudhuri, A. Hadjadj, A. Chinnayya, On the use of immersed boundary methods for shock/obstacle interactions, J. Comput. Phys. 230 (2011) $1731-1748$.

[27] M. Wu, W. Kuo, Accelerative expansion and ddt of stoichiometric ethylene/oxygen flame rings in micro-gaps, Proc. of the Combust. Inst. 34 (2013) 2017-2024. 


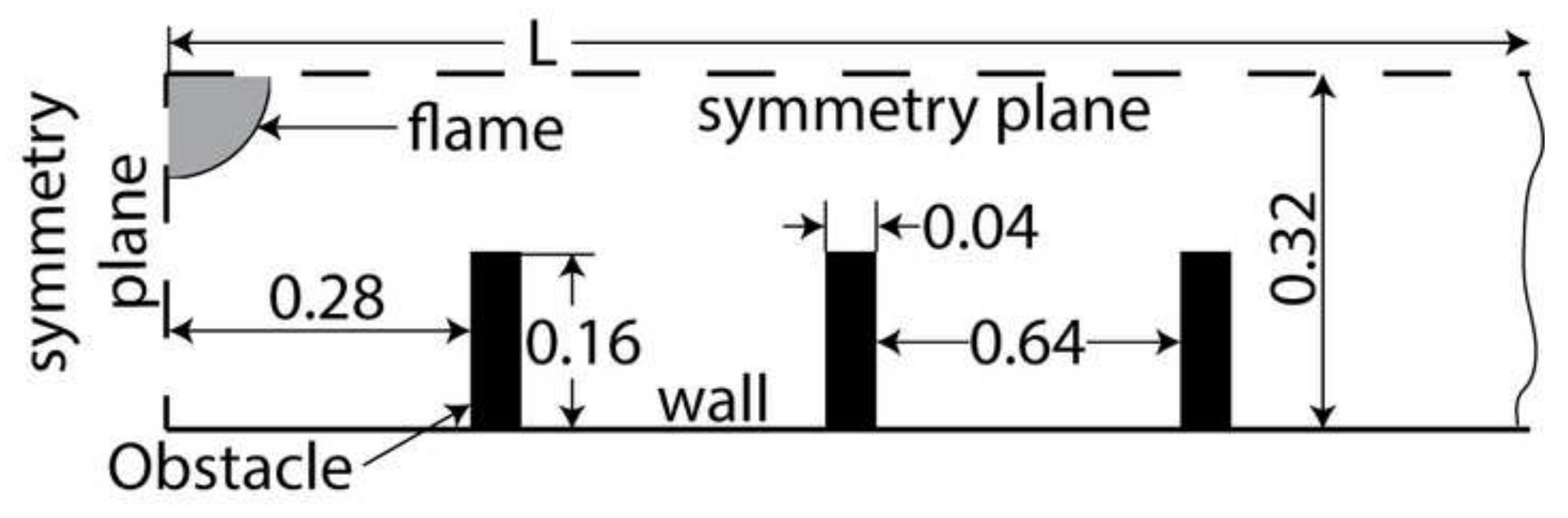




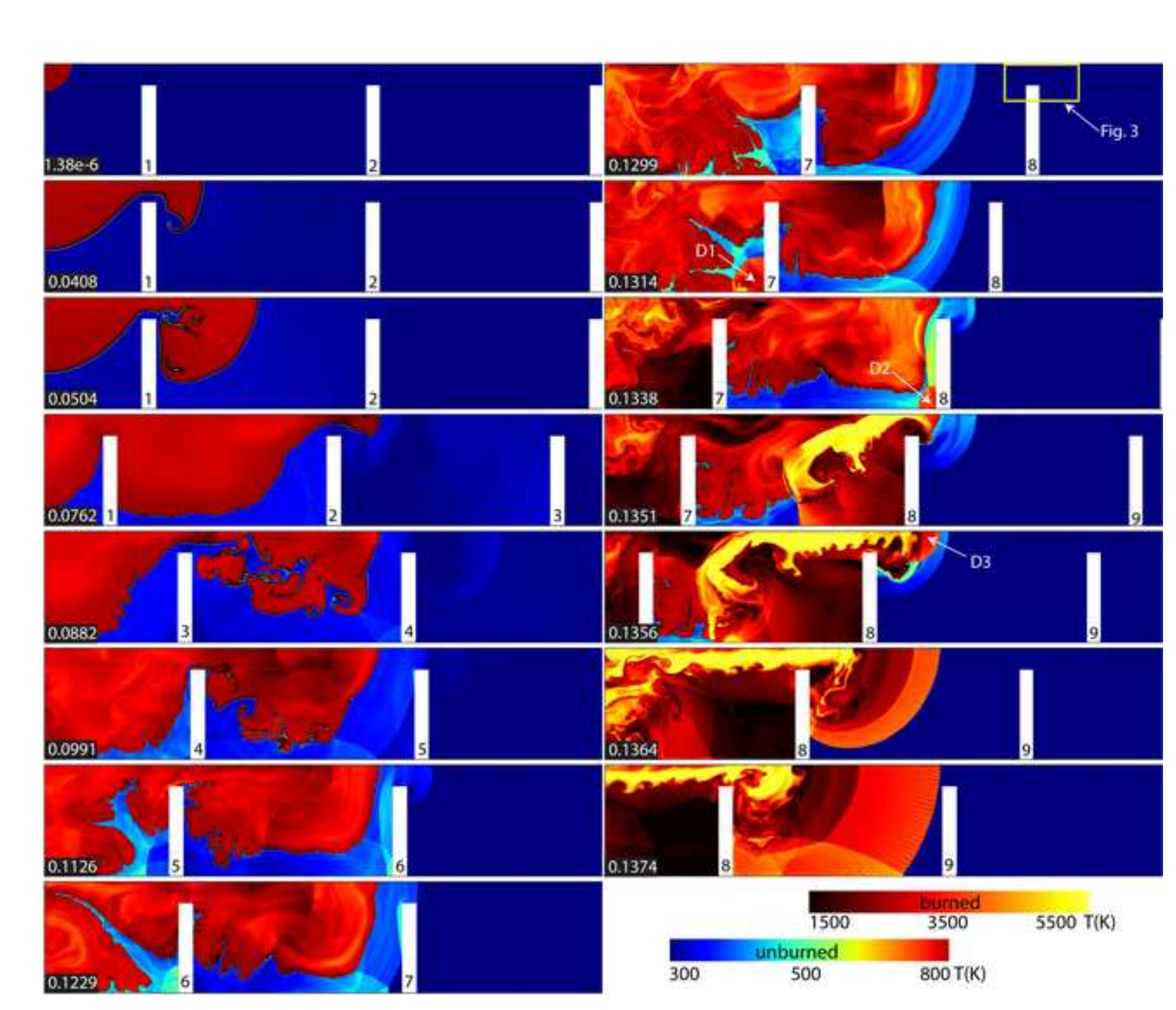

\subsection{8}

0.0504

0.0762

列

Thenest 


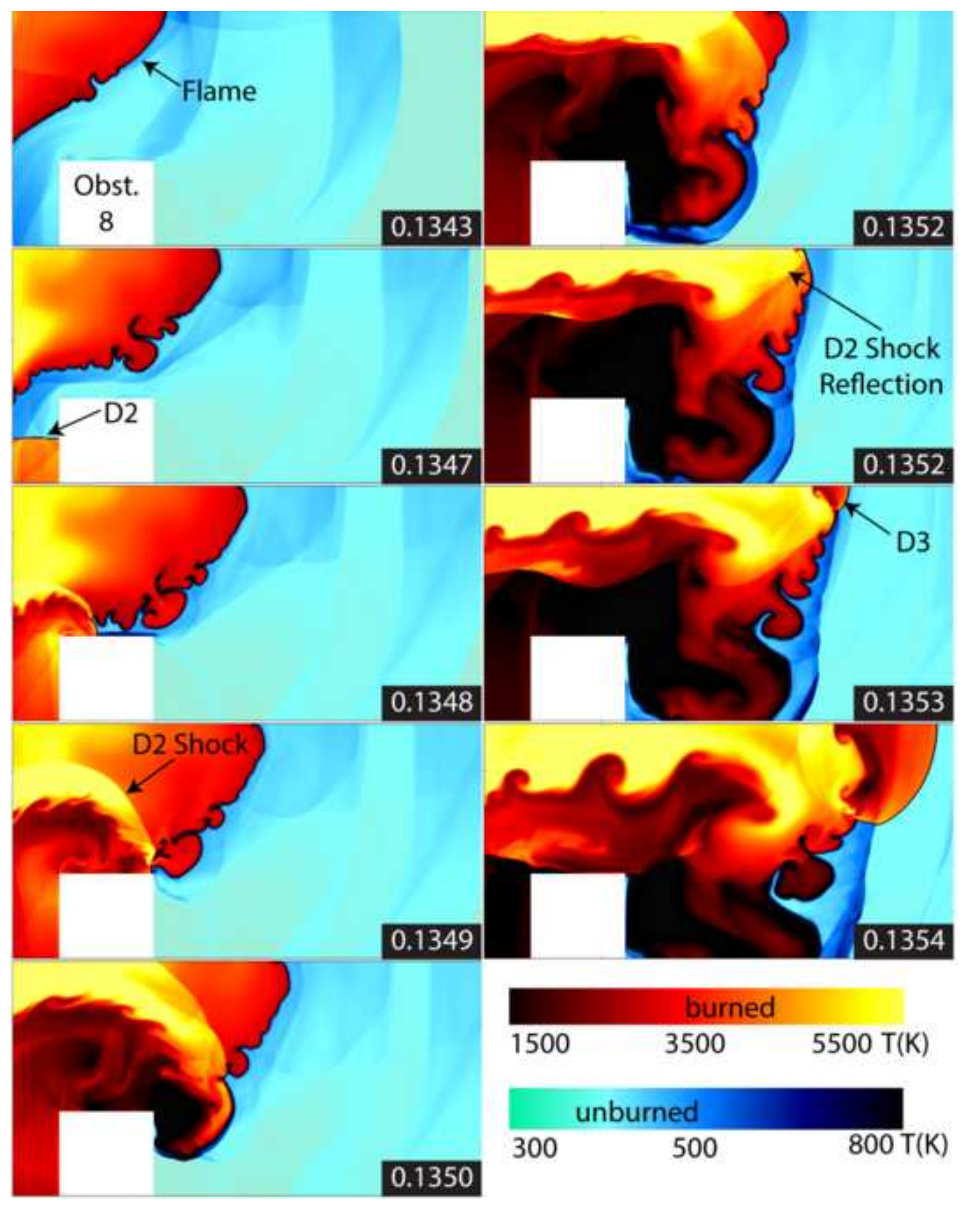

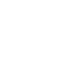

更$$
\text { (1) }
$$ 


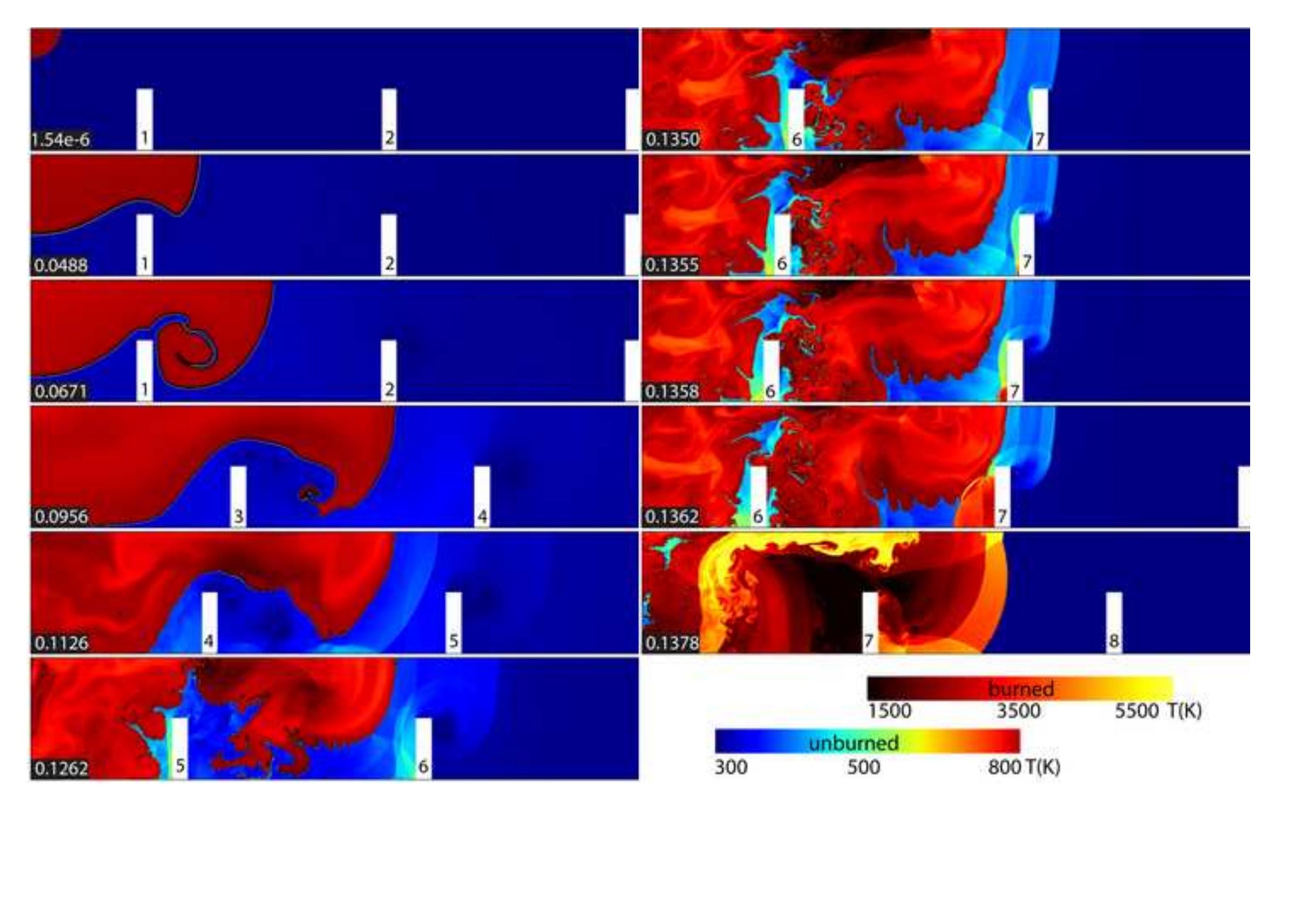

$$
300 \quad 500 \quad 800 \mathrm{~T}(\mathrm{~K})
$$



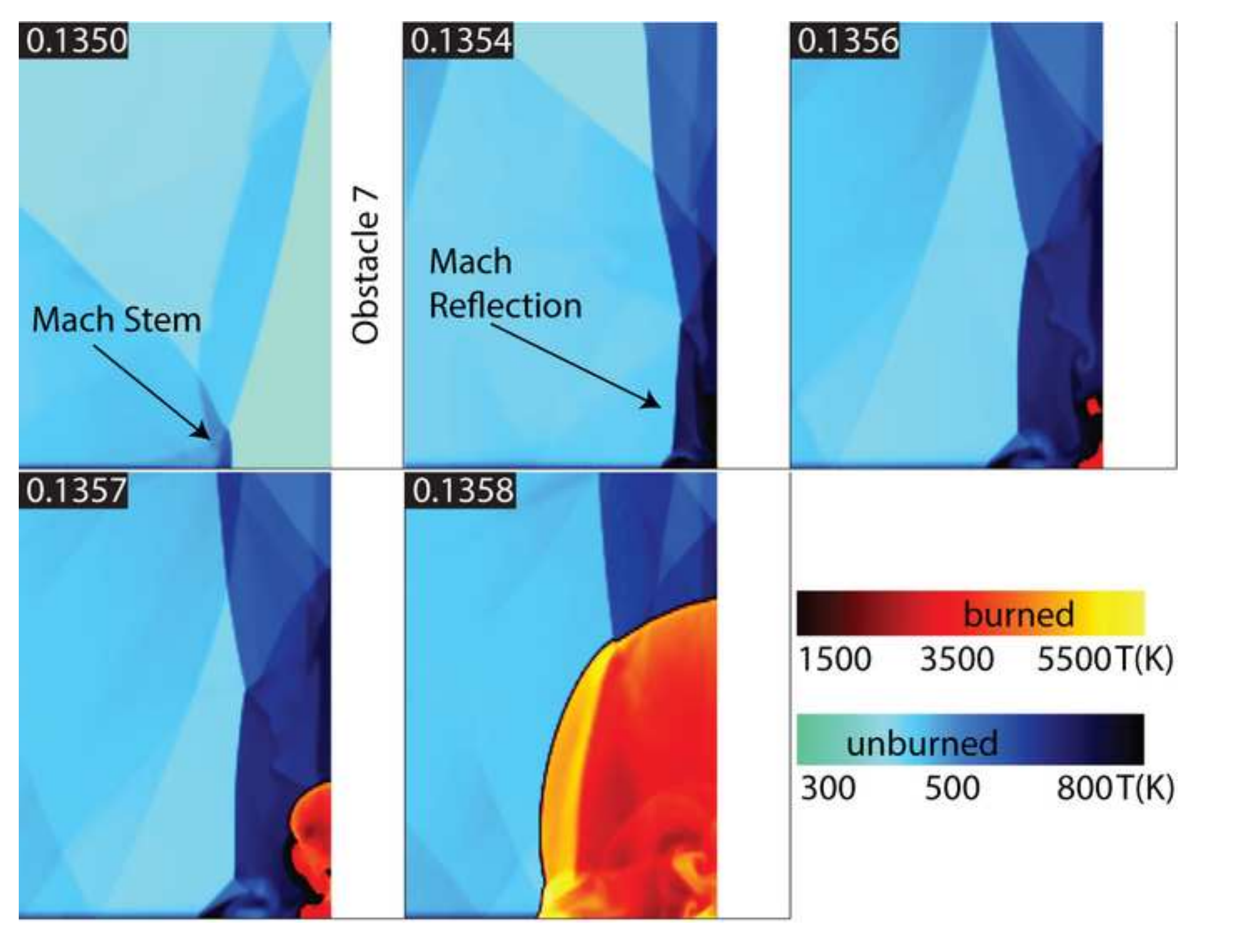
0.1804

a.

0.248

0.2558

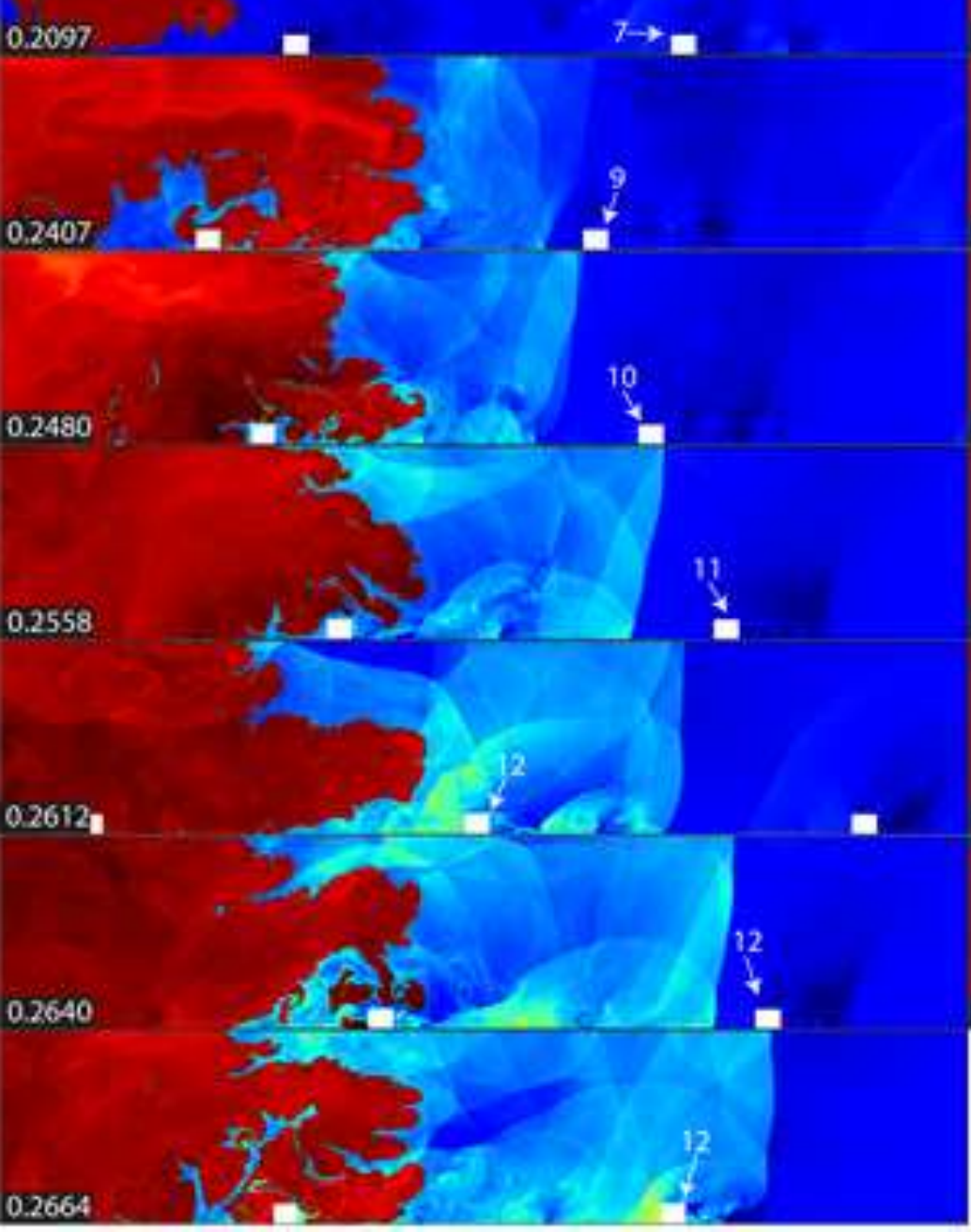

0.2687

. 0.2690
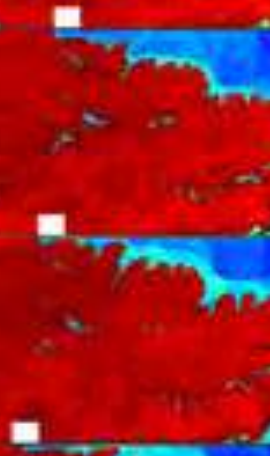

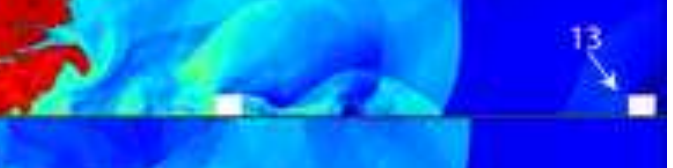

0.2699

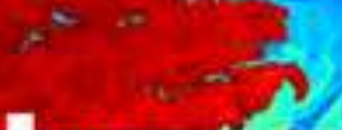

$1^{13}$

0.2707

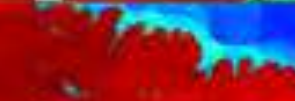

694 무
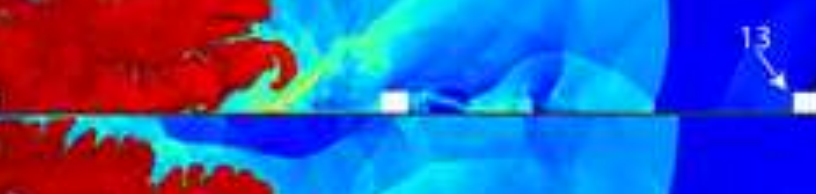

0.2708
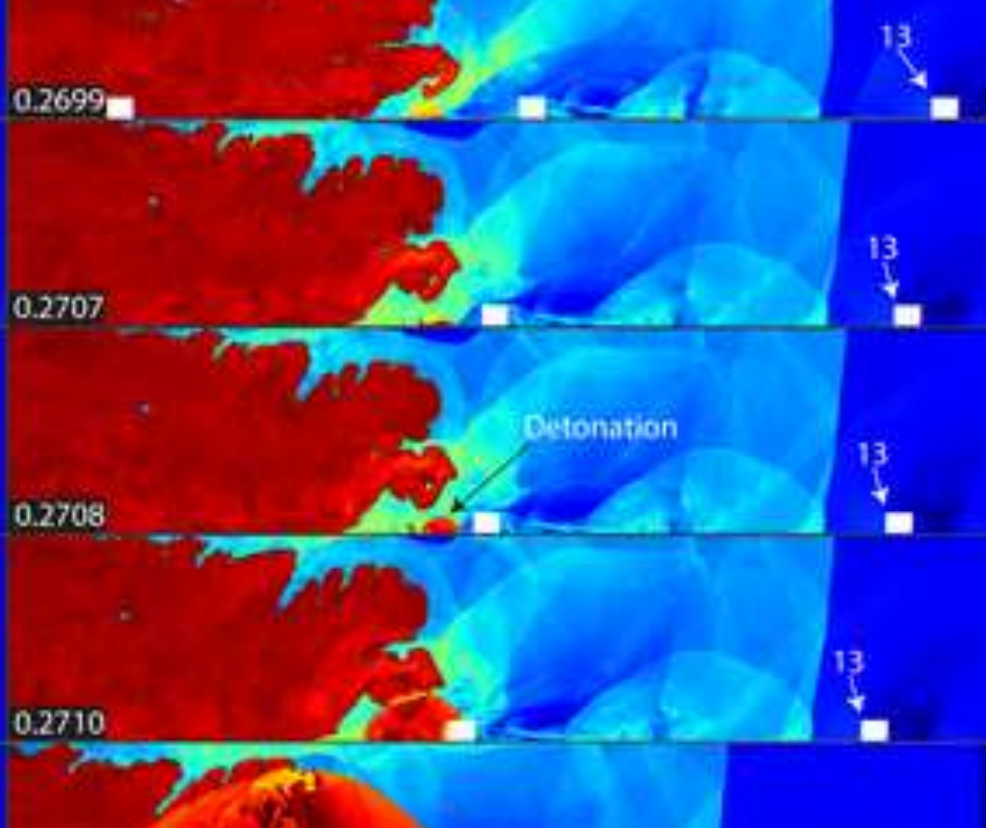
0.2719
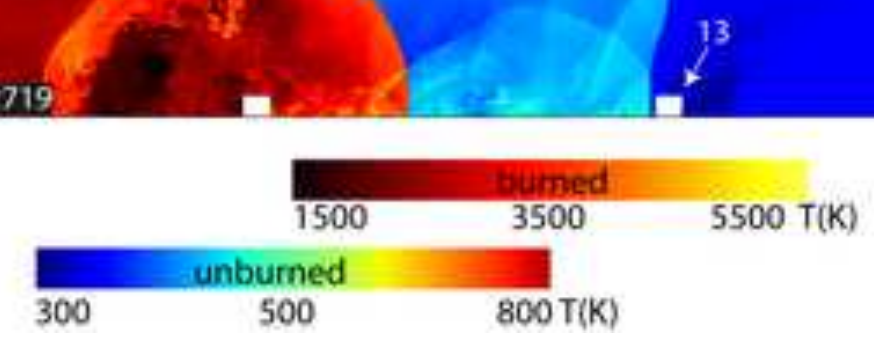

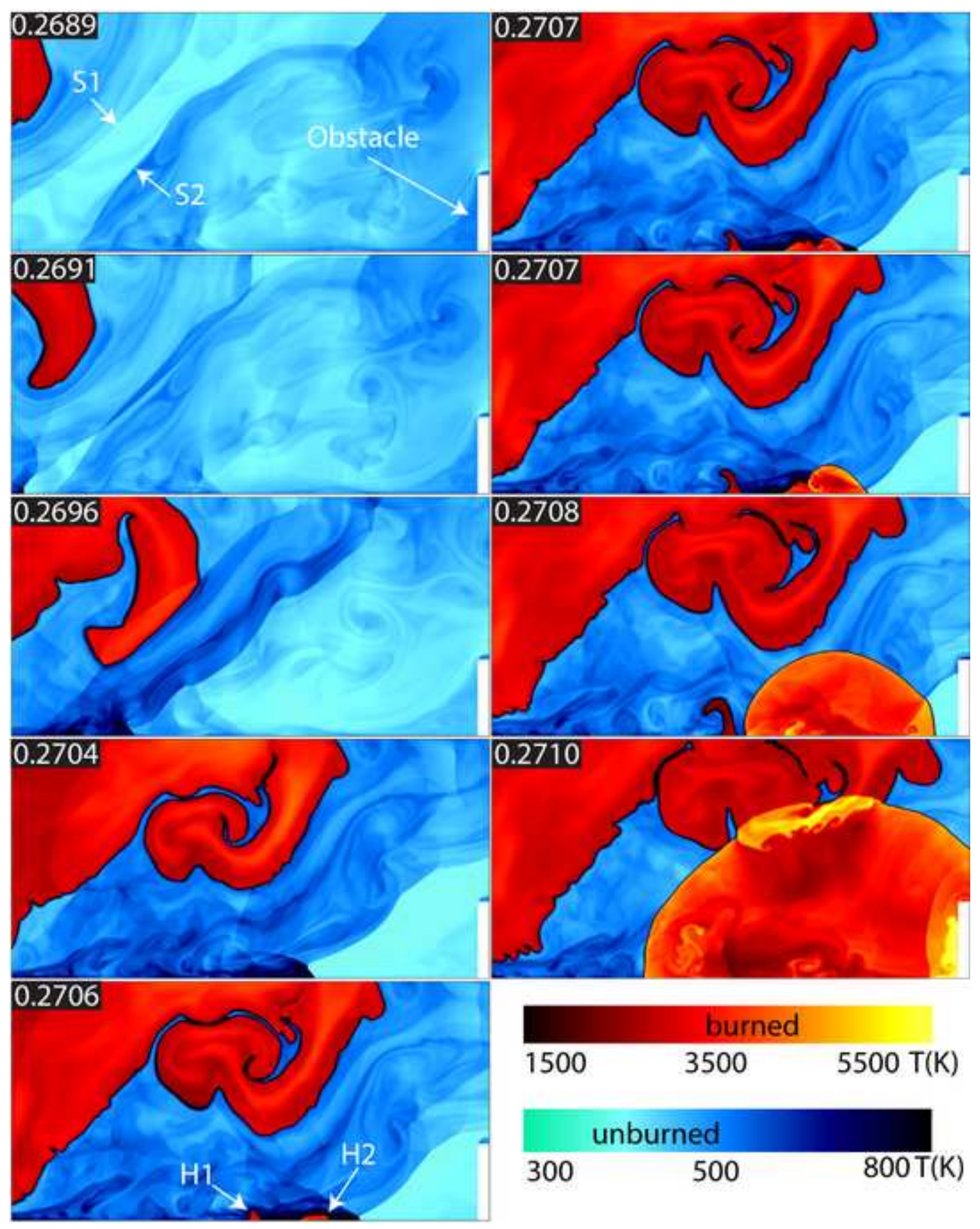

burned

\begin{tabular}{lrr}
\hline 1500 & 3500 & $5500 \mathrm{~T}(\mathrm{~K})$ \\
& & \\
300 & 500 & $800 \mathrm{~T}(\mathrm{~K})$
\end{tabular}




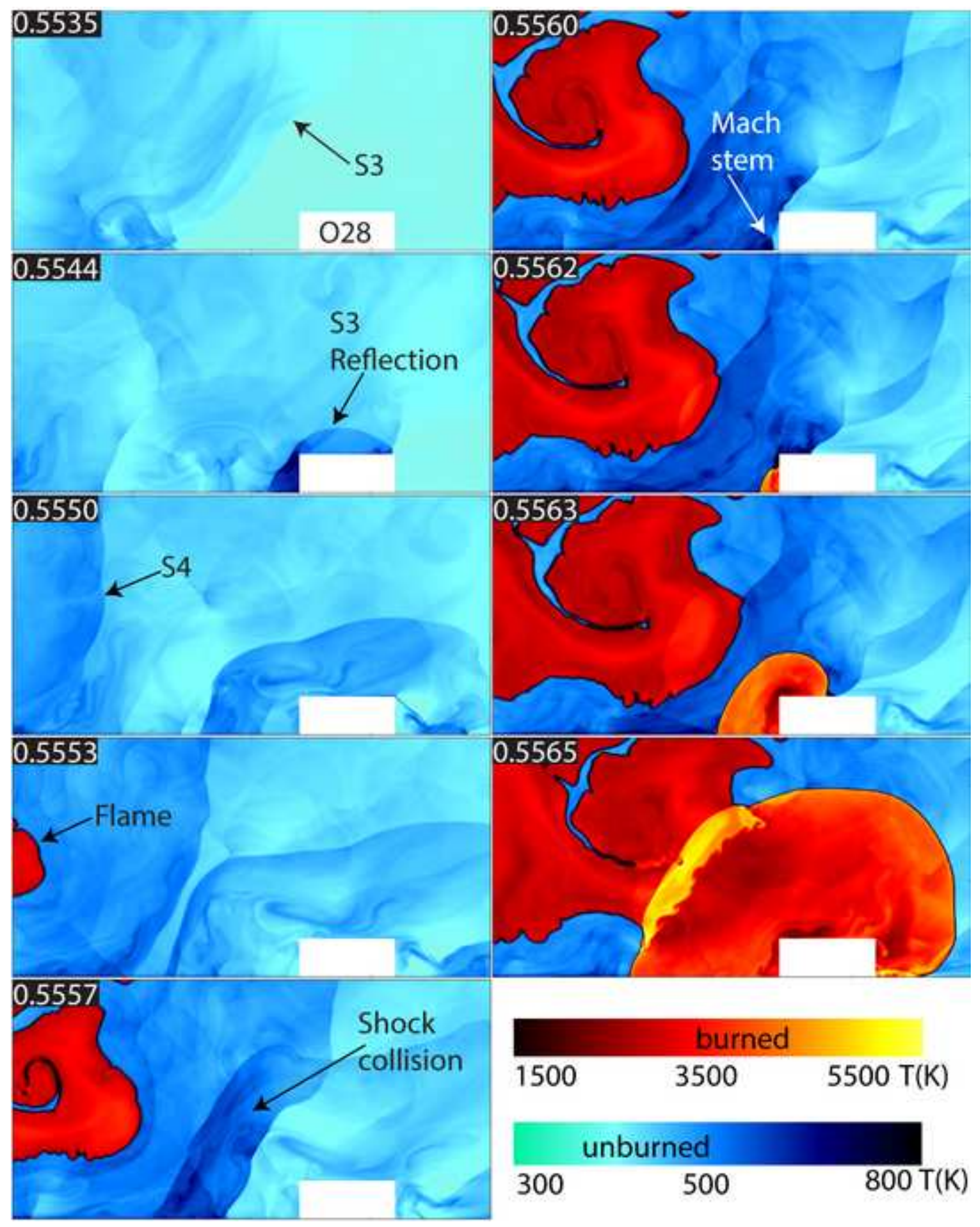




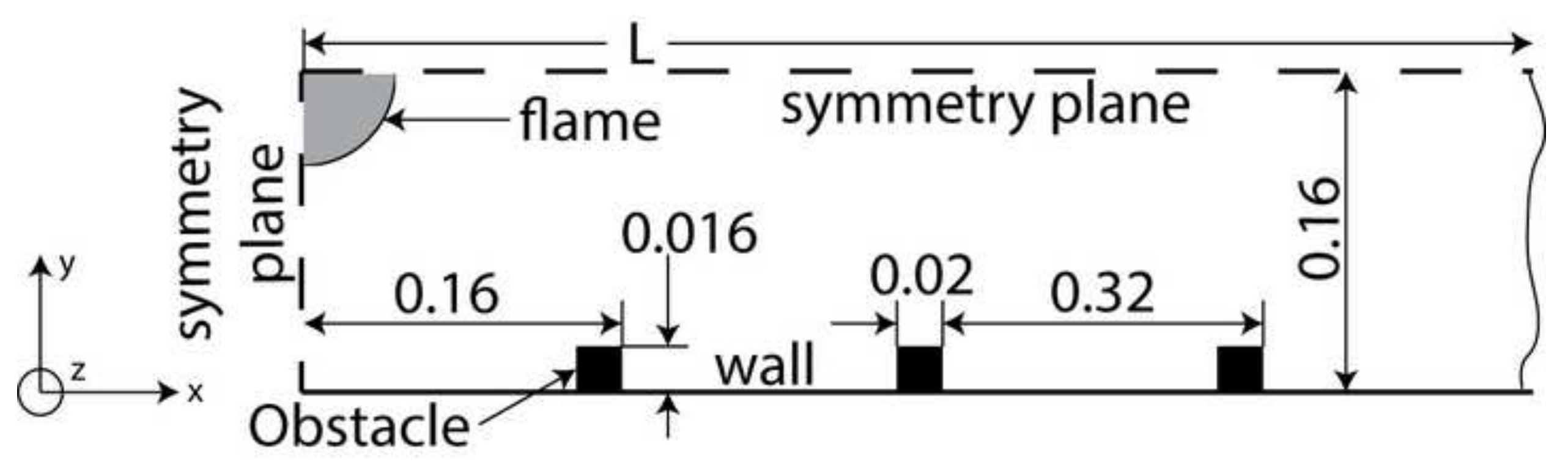




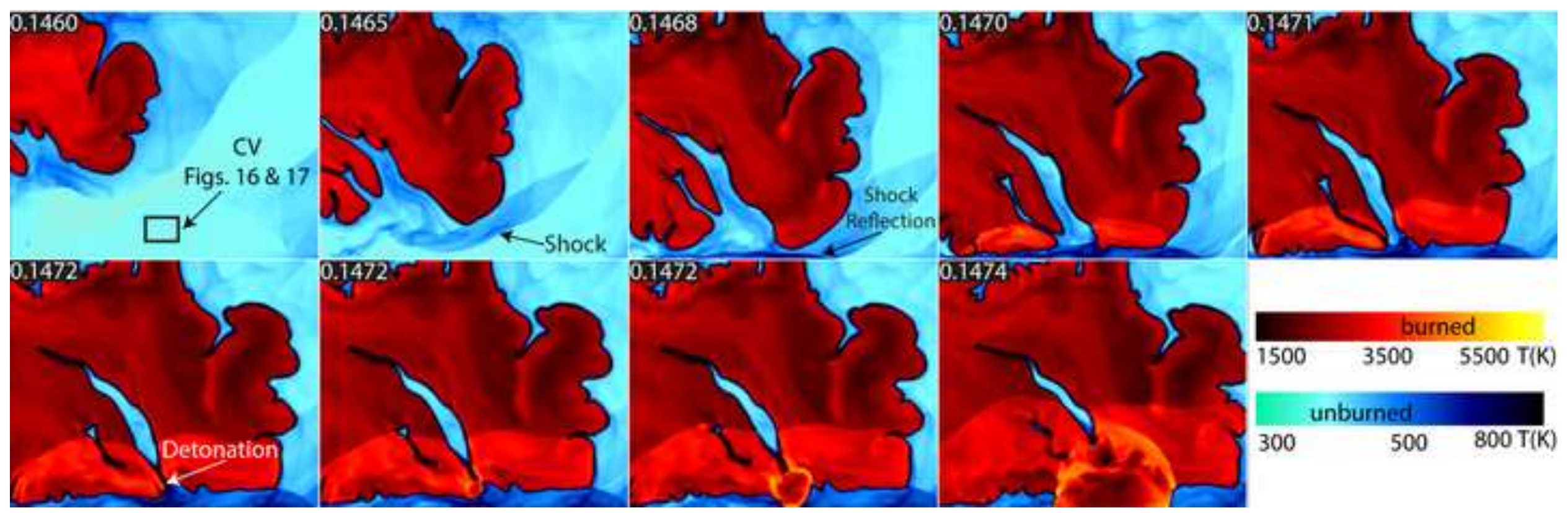



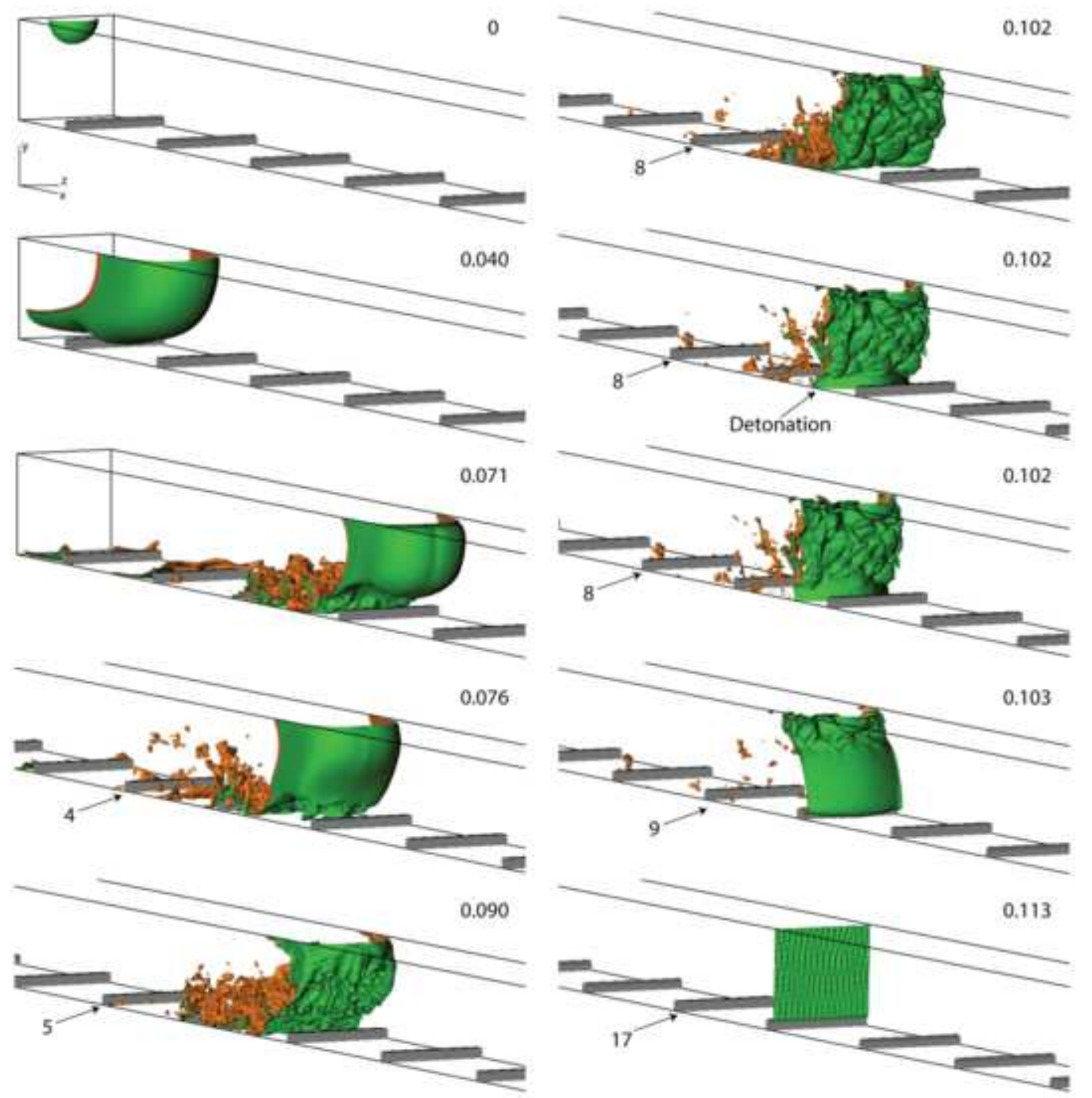


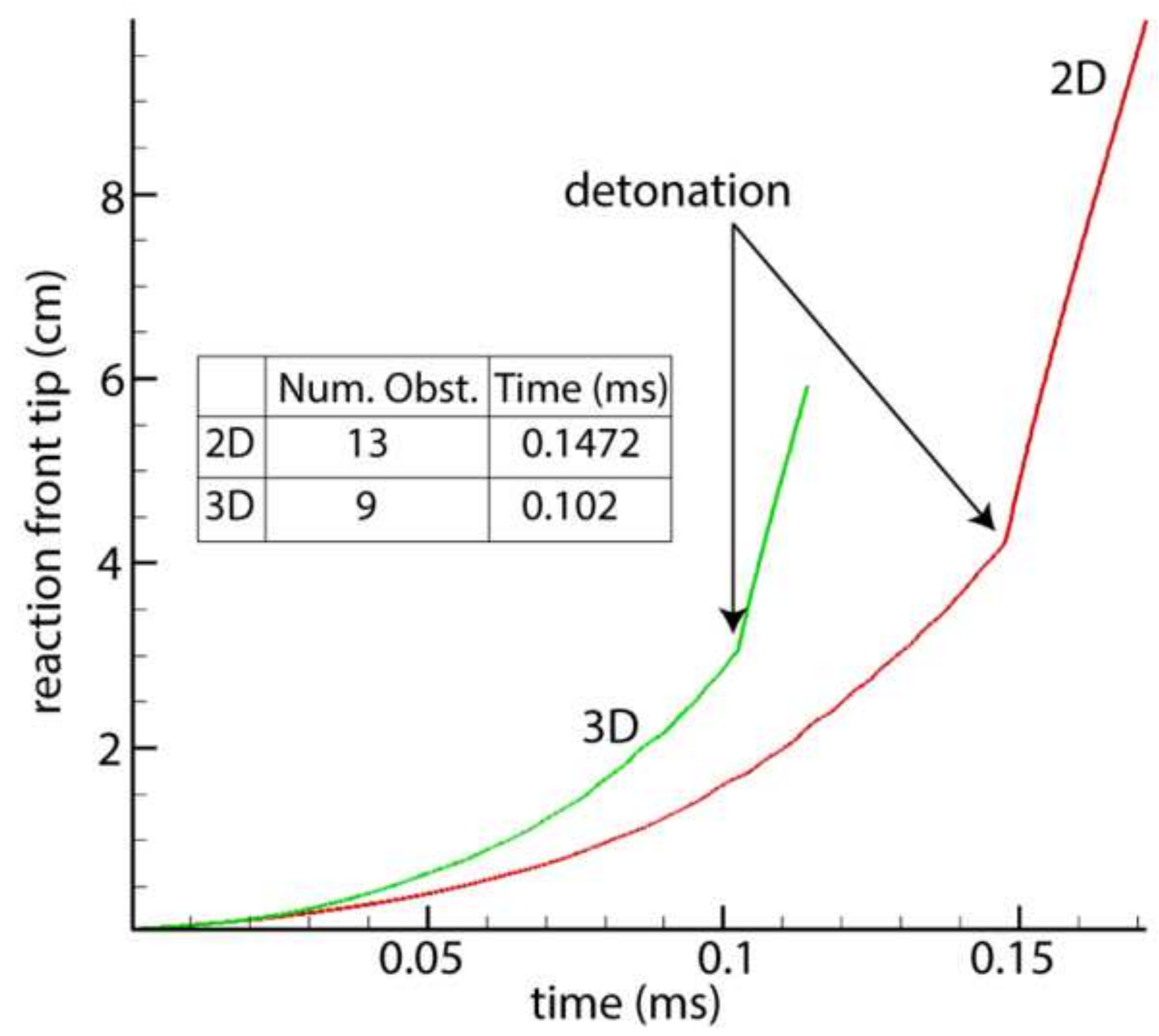




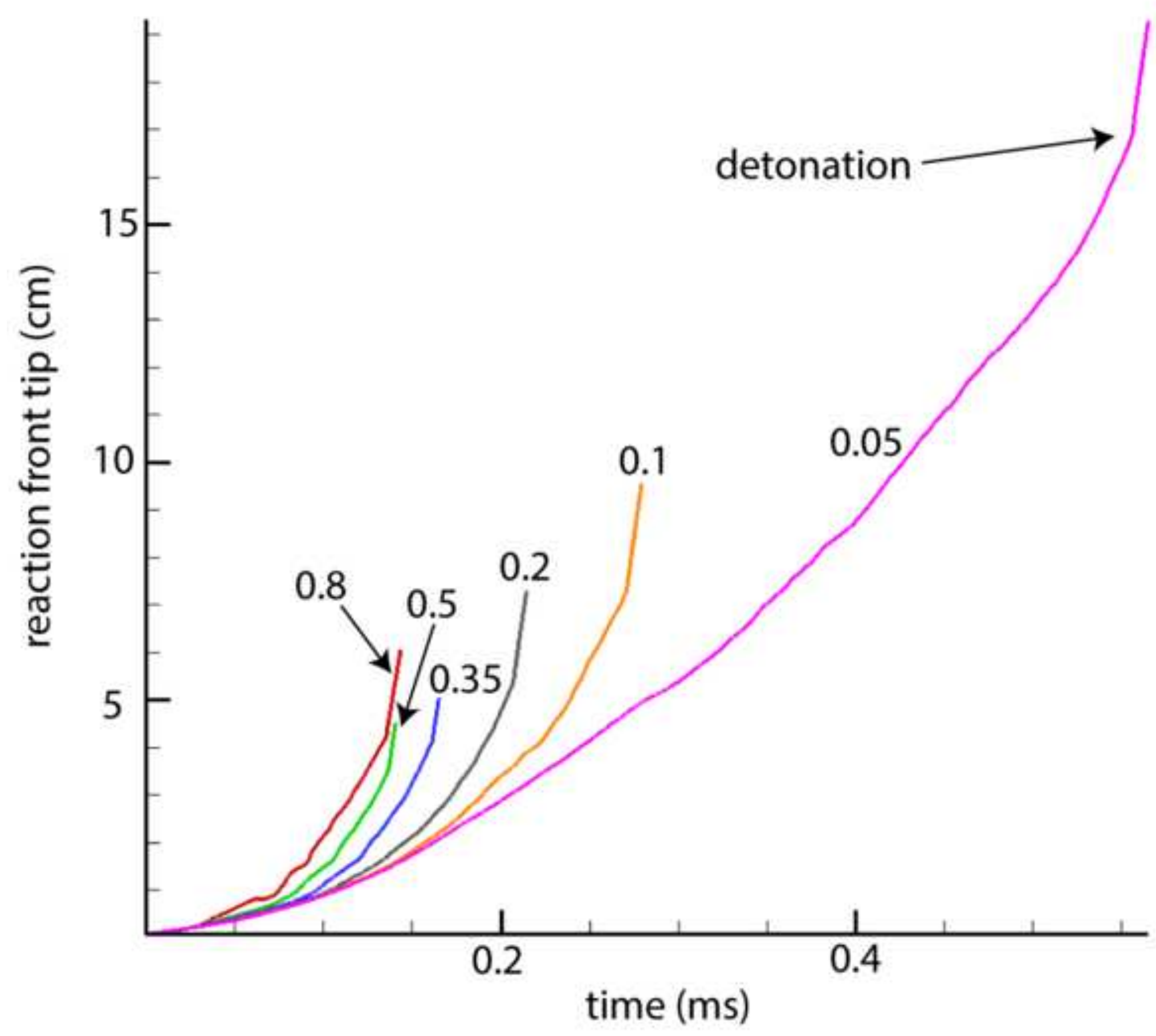




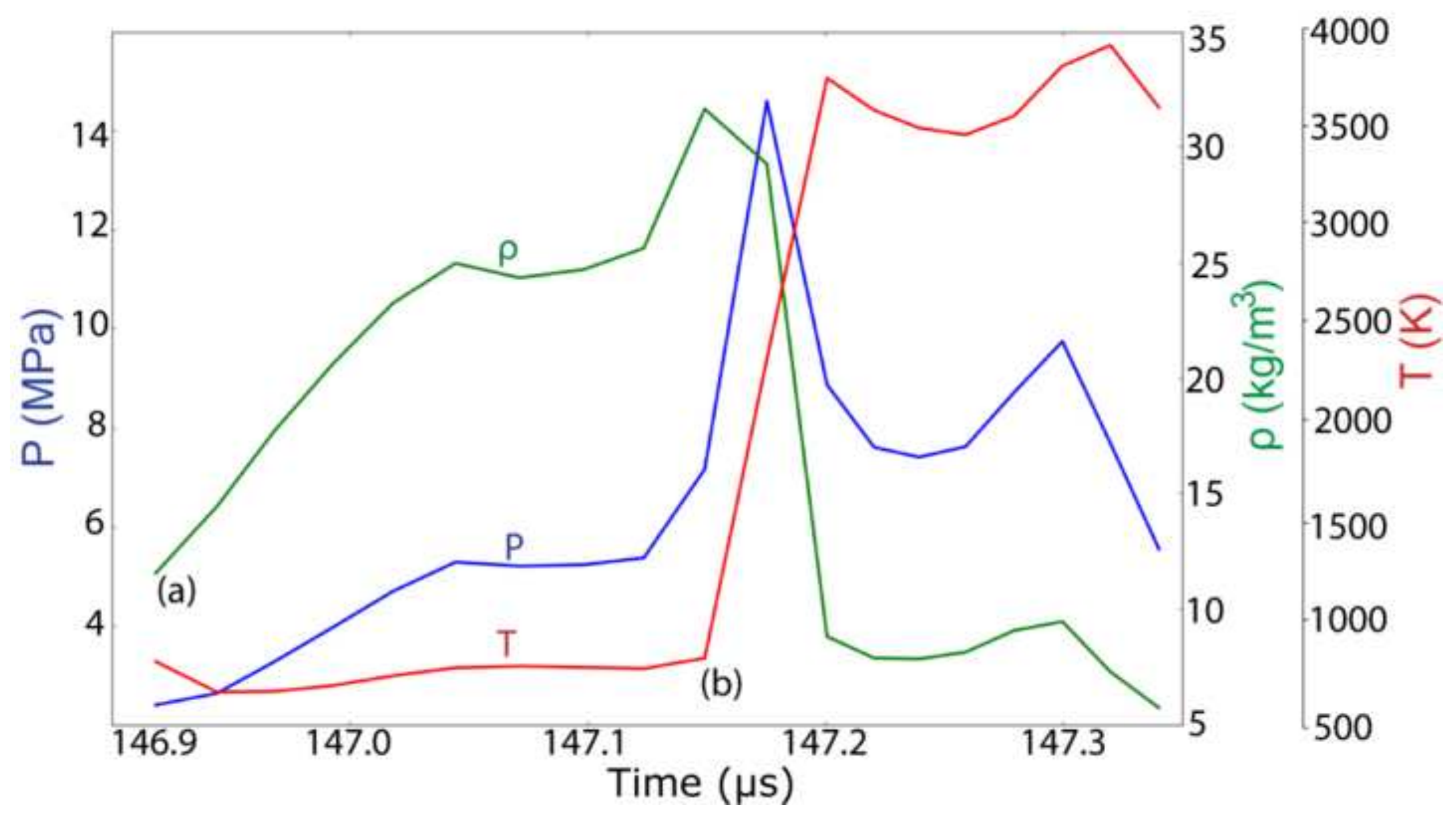




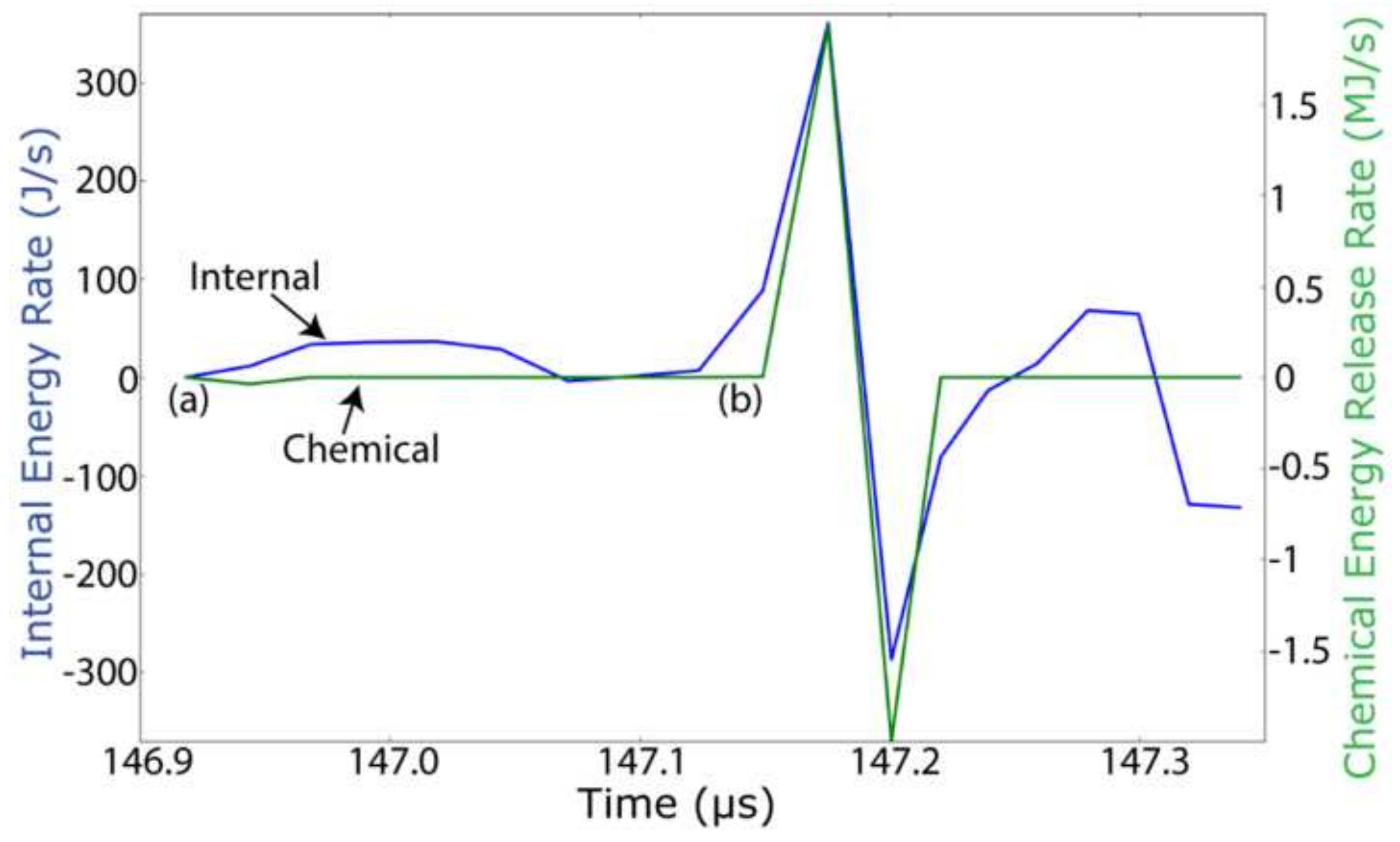




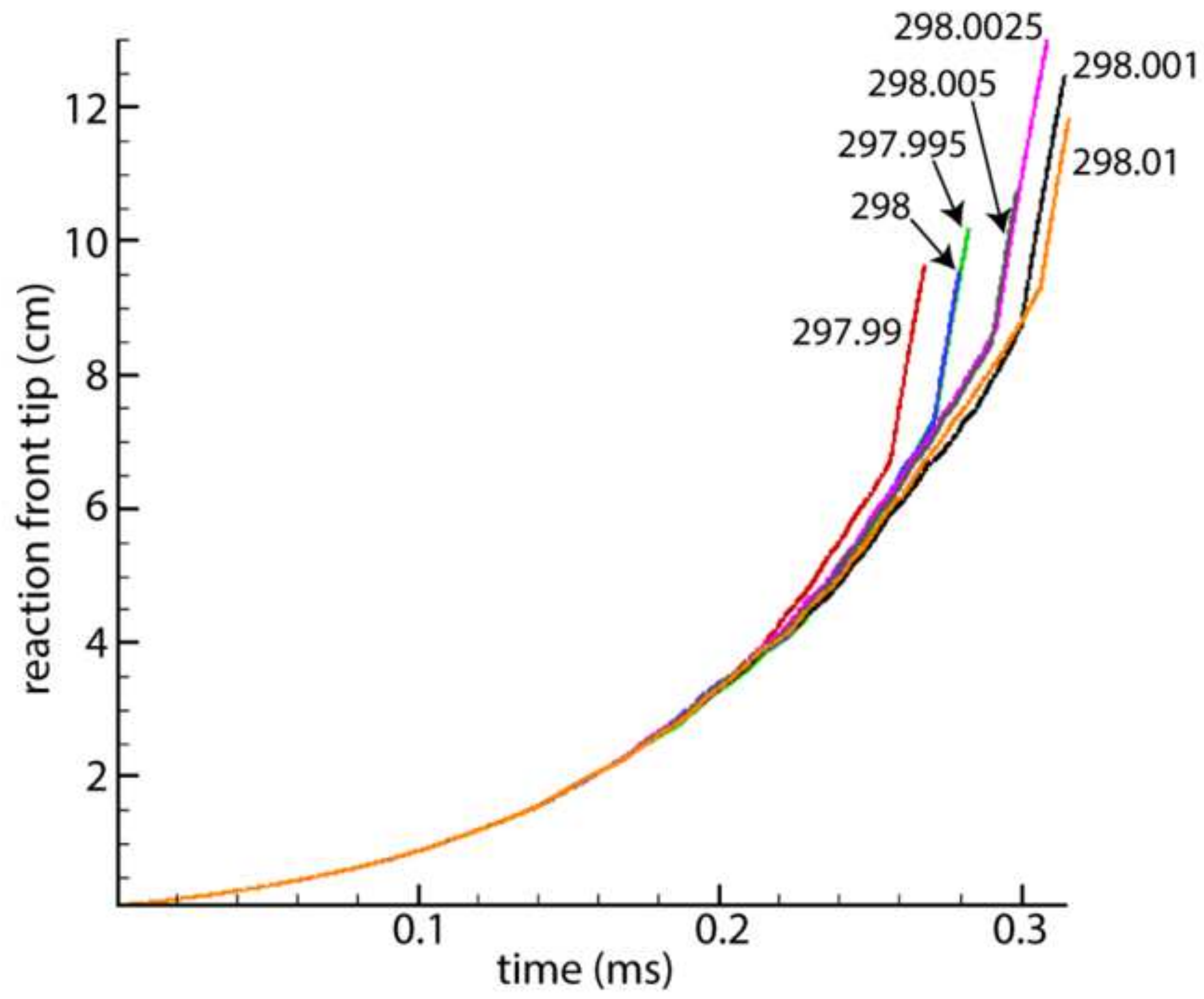



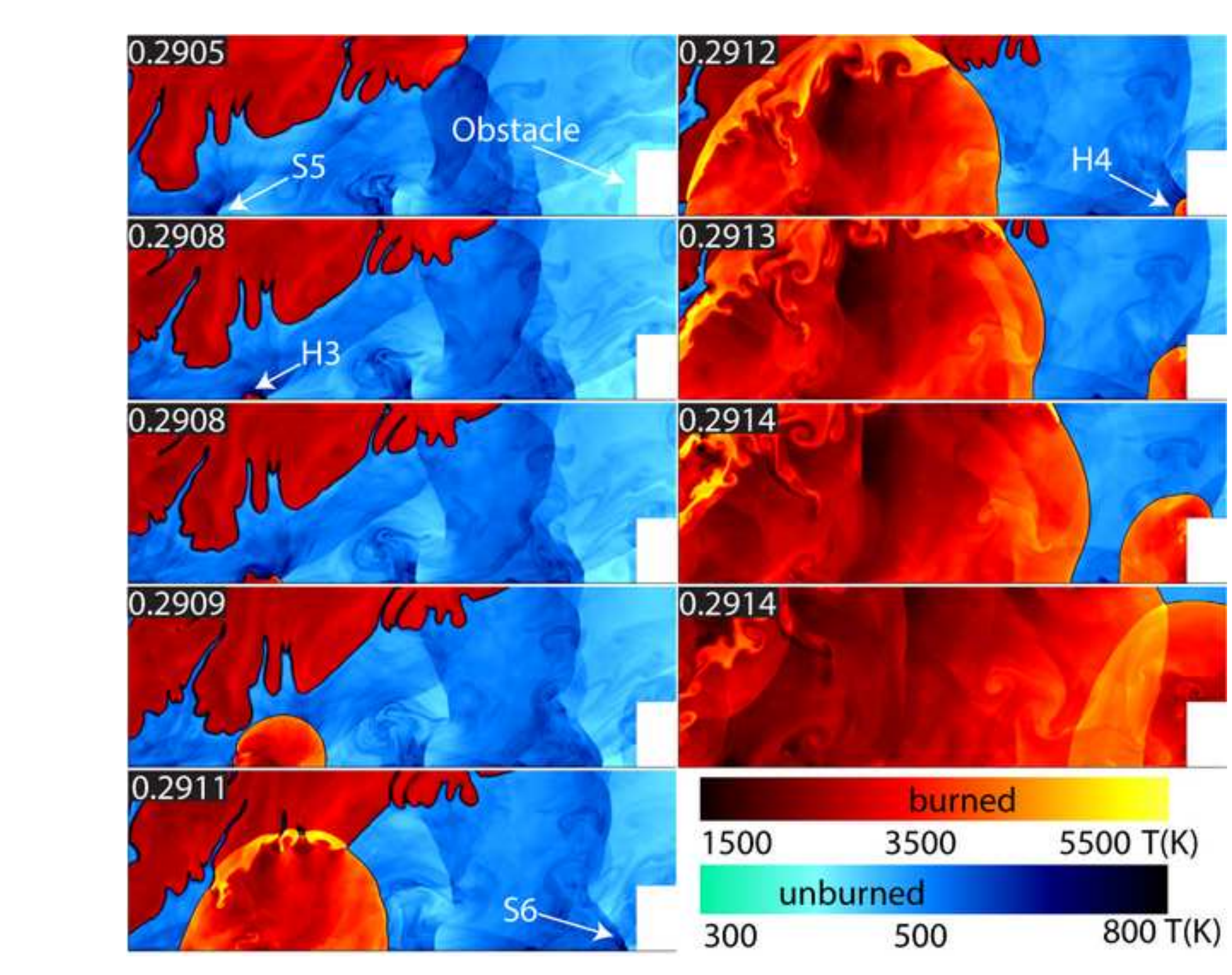

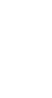

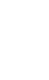

.

